\title{
Propagation of Chaos in a Coagulation Model
}

June 18, 2012

\author{
M. Escobedo ${ }^{1,2}$ and F. Pezzotti ${ }^{3}$
}

Abstract. The dynamics of a finite system of coalescing particles in a finite volume is considered. It is shown that, in the thermodynamic limit, a coagulation equation is recovered and propagation of chaos holds for all time.

Key words. Coagulation equation, BBGKY hierarchy, propagation of chaos.

\section{Introduction}

Coagulation equations are widespread models describing large sets of coalescing particles. Each particle is characterised by a single non negative real number $m$, that we call "mass" although it can be a mass, a length, a charge, etc... It is argued in such descriptions that the coagulation rate depends on the masses of the particles. In the spatially homogeneous models it is further assumed that spatial fluctuations in the mass density are negligible. In the first example of this sort, proposed by Smoluchowski [55], particles of radius $r$ moving by Brownian motion with variance proportional to $1 / r$ meet at rate proportional to $\left(r_{1}+r_{2}\right)\left(r_{1}^{-1}+r_{2}^{-1}\right)$. The concentration $f(t, m)$ of particles of mass $m=1,2,3, \cdots$ at time $t$ satisfies the equation:

$$
\begin{aligned}
& \frac{\partial f}{\partial t}(t, m)=\frac{1}{2} \sum_{k=1}^{m-1} A(m-k, k) f(t, m-k) f(t, k)-f(t, m) \sum_{k=1}^{\infty} A(m, k) f(t, k), \\
& A(m, k)=\left(m^{1 / 3}+k^{1 / 3}\right)\left(m^{-1 / 3}+k^{-1 / 3}\right) .
\end{aligned}
$$

If masses are assumed to take continuous, positive values we are led to the continuous version:

$$
\frac{\partial f}{\partial t}(t, m)=\frac{1}{2} \int_{0}^{m} A\left(m-m^{\prime}, m^{\prime}\right) f\left(t, m-m^{\prime}\right) f\left(t, m^{\prime}\right) d m^{\prime}-f(t, m) \int_{0}^{\infty} A\left(m, m^{\prime}\right) f\left(t, m^{\prime}\right) d m^{\prime} .
$$

Different coagulation kernels $A\left(m, m^{\prime}\right)$ may be considered. In particular constant, additive and multiplicative kernels have been widely studied. The concentration $f(t, m)$ is defined as the average

\footnotetext{
${ }^{1}$ Departamento de Matemáticas, Universidad del País Vasco (UPV/EHU), Apartado 644, E-48080 Bilbao, Spain. E-mail: miguel.escobedo@ehu.es

${ }^{2}$ Basque Center for Applied Mathematics (BCAM), Alameda Mazarredo 14, 48009 Bilbao, Spain.

${ }^{3}$ Dipartimento di Matematica "G. Castelnuovo", Università di Roma "La Sapienza", P.le Aldo Moro 5, 00185 Roma, Italy. E-mail: pezzotti@mat.uniroma1.it
} 
number of particles of mass $m$ per unit volume at time $t$ in the discrete case, and as the average number of particles of mass in $[m, m+d m]$ per unit volume at time $t$ in the continuous case.

Coagulation equations are used in a variety of fields like polymer science, atmosphere physics, astrophysics and colloidal chemistry, to model large systems of particles that collide and coalesce (see [2], [16], [23], [40] and references therein.). The coagulation operator, in the right hand side of (1.1), also appears in mathematical models of biology and immunology to describe conservative interactions between different entities in complex biologic systems (see [4], [6], [7], [8], [9], [36] and references therein). Equation (1.1) considers only one single type of particles and does not take into account other possible interactions like, for example, fragmentation, proliferation or destruction. These effects, and others, are considered in more general models, introduced in physics, biology and medicine, to describe large systems of interacting entities. A general class of multilinear Boltzmann-like equations describing the dynamics of individuals undergoing kinetic interactions is introduced in [35], [36], [37]. General systems of Boltzmann-type equations are considered in [4], [5], [6], [7], [8] and [9] for the modeling of complex biological systems.

On the other hand, a stochastic process, that describes the dynamics of a finite particle system undergoing aggregation by binary collisions, and is related to the coagulation equation (1.1), was introduced in a general context by several authors as in [25], [42] and [43] (see also [25], [52] for coalescing droplets and [56] in astrophysics) and is now currently known as Marcus-Lushnikov process.

When the number of coalescing particles in the finite system is very large, it is reasonable to think that the dynamics induced by the coagulation equation and the Marcus-Lushnikov process may be close. In particular, one may try to derive the solution of the coagulation equation as limit, in some suitable sense, of the solution of the Marcus-Lushnikov process. Such convergence result, under suitable hypothesis for the initial data, is sometimes known as propagation of chaos. This question is related of course with one basic issue in kinetic theory: the connection between one-particle effective descriptions and their corresponding many-body dynamics.

Beside the derivation of the coagulation equation, finite systems of particles play an important role in numerical algorithms (see for example the review [51]). Many stochastic algorithms for equation (1.1) are based on the Marcus-Lushnikov process (cf. [14], [19], [24], [25], [26], [29], [51]). Convergence properties of these algorithms have been derived from [18], [28], [33], [47]. Another stochastic process was introduced in [20] in the context of numerical treatment of the coagulation equation (1.1). A different finite system, that also leads to equation (1.1), was introduced in [35], [36], [37], mainly motivated by medical and biological processes.

Our purpose in this work is to prove, using classical methods of kinetic theory, that propagation of chaos holds for the finite system of particles whose dynamics is given by the Marcus-Lushnikov process, and to show that equation (1.1) describes asymptotically the time evolution of the particle number density of such a system.

To this end, we consider, in a given volume $V$, a finite system of particles whose number $N$ changes with time, due to the coagulation process. Particles are assumed to collide and coalesce, at a certain rate $A$. Binary collisions are dominant and therefore collisions of three or more particles are neglected. Finally, the coalescence mechanism preserves the total mass of the two coalescing particles. At all time $t \geq 0$, for any $N \in \mathbb{N}^{*}$ and $\left(m_{1}, m_{2}, \cdots, m_{N}\right) \in\left(\mathbb{R}^{+}\right)^{N}$ we consider the mass distribution functions $P_{N}\left(t, m_{1}, \ldots, m_{N}\right)$. Each of these functions describe the state of a system constituted by $N$ particles in a volume $V$ where, at time $t$, one particle has mass between $m_{1}$ and $m_{1}+d m_{1}$, one has 
mass between $m_{2}$ and $m_{2}+d m_{2}$ and so on. We may then define the probability to have $N$ particles in the volume $V$ at time $t=0$ as follows:

$$
P(0, N)=\frac{1}{N !} \int_{0}^{\infty} \cdots \int_{0}^{\infty} P_{N}^{0}\left(m_{1}, \ldots, m_{N}\right) d m_{1} \cdots d m_{N},
$$

and then:

$$
\sum_{N=1}^{\infty} P(0, N)=1
$$

Given any $N_{0} \in \mathbb{N}^{*}$ we consider initial data satisfying the following conditions:

(i) $P_{N}^{0}\left(m_{1}, \ldots, m_{N}\right)=0$, for all $N \neq N_{0}$,

(ii) $P_{N_{0}}^{0}\left(m_{1}, \ldots, m_{N_{0}}\right)=\left(N_{0}\right) ! f_{0}\left(m_{1}\right) \otimes \cdots \otimes f_{0}\left(m_{N_{0}}\right), m_{i} \in(0,+\infty), \quad i=1,2, \ldots N_{0}$

$$
\text { where: } \quad f_{0} \geq 0, \int_{0}^{\infty} f_{0}(m) d m=1
$$

Condition $(i)$ expresses that at $t=0$ the system has exactly $N_{0}$ particles. It is easily seen that conditions $(i)$ and $(i i)$ imply $P(0, N)=\delta\left(N-N_{0}\right)$. The average number of particles is then:

$$
\sum_{N=1}^{\infty} N P(N, 0)=N_{0}
$$

as expected.

The starting point of our analysis is the set of evolution equations satisfied by the mass distribution functions $P_{N}\left(t, m_{1}, \ldots, m_{N}\right)$ throughout the coagulation process. These equations where obtained in [43] and later in [25], [41], [56] in the study of the Marcus-Lushnikov process. It is possible to deduce from these equations a new system of equations for the correlation functions:

$$
f_{j}\left(m_{1}, \ldots, m_{j}, t\right)=\sum_{N=j}^{\infty} \frac{1}{(N-j) !} \int_{0}^{\infty} d m_{j+1} \ldots \int_{0}^{\infty} d m_{N} P_{N}\left(m_{1}, \ldots, m_{N}, t\right),
$$

where $j \in \mathbb{N}^{*}$. Notice that for $j=1$ we have

$$
\begin{aligned}
\bar{N}(t) & =\sum_{N=1}^{\infty} N P(t, N)=\sum_{N=1}^{\infty} \frac{1}{(N-1) !} \int_{0}^{\infty} \cdots \int_{0}^{\infty} P_{N}\left(t, m_{1}, m_{2}, \cdots, m_{N}\right) d m_{1} \cdots d m_{N} \\
& =\int_{0}^{\infty} f_{1}\left(t, m_{1}\right) d m_{1},
\end{aligned}
$$

and therefore $f_{1}(t)$ is the density function associated to the mean number of particles at time $t$. 
We define now the rescaled functions:

$$
f_{j}^{V}\left(t, m_{1}, \cdots, m_{j}\right)=\frac{f_{j}\left(t, m_{1}, \cdots, m_{j}\right)}{V^{j}},
$$

and study their limit as

$$
N_{0} \rightarrow+\infty, V \rightarrow+\infty, \frac{N_{0}}{V} \rightarrow \rho_{0}
$$

Notice that, for $j=1$ :

$$
\int_{0}^{\infty} f_{1}^{V}\left(t, m_{1}\right) d m_{1}=\frac{\overline{N(t)}}{V}=\text { mean number of particles per unit volume at time } t \geq 0 .
$$

By (1.2), we have

$$
f_{j}^{V}\left(0, m_{1}, \cdots, m_{j}\right)=\frac{N_{0}\left(N_{0}-1\right) \ldots\left(N_{0}-j+1\right)}{V^{j}} f_{0}^{\otimes j}\left(m_{1}, \cdots, m_{j}\right),
$$

and it easily follows that:

$$
\forall j \in \mathbb{N}^{*}: \lim _{\substack{V, N_{0} \rightarrow+\infty \\ \frac{N_{0}}{V} \rightarrow \rho_{0}}}\left\|f_{j}^{V}(0)-\left(\rho_{0}\right)^{j} f_{0}^{\otimes j}\right\|_{L^{1}\left(\left(\mathbb{R}^{+}\right)^{j}\right)}=0 .
$$

The main result of the paper is the following.

Theorem 1.1 Suppose that the coagulation kernel $A$ is bounded. Let $\left\{f_{j}^{V}(t)\right\}_{j \in \mathbb{N}^{*}}$ be the sequence of functions defined in (1.4). Then,

$$
\forall j \geq 1: \quad \lim _{\substack{V, N_{0} \rightarrow+\infty \\ \frac{N_{0}}{V} \rightarrow \rho_{0}}}\left\|f_{j}^{V}(t)-f(t)^{\otimes j}\right\|_{L^{1}\left(\left(\mathbb{R}^{+}\right)^{j}\right)}=0,
$$

where $f$ is the unique solution in $\mathbf{C}\left([0,+\infty) ; L^{1}\left(\mathbb{R}^{+}\right)\right)$of the coagulation equation (1.1) with initial datum $\rho_{0} f_{0}$.

The convergence result (1.8) under the hypothesis (1.6) for the initial data is usually known as propagation of chaos. By the initial condition (1.6) the particles are identically and independently distributed at time $t=0$. The conclusion (1.8) means that at any time $t>0$, the system of particles still satisfies that property but only asymptotically, in the limit (1.5) (cf. [49] and references therein). The results previously known stated that this approximation was true in some weaker topology (see [22], [33], [34], [47]).

From the point of view of applications, we may describe the consequence of our main result as follows. The coagulation equation (1.1) is used as a mathematical model to describe large systems of coalescing entities in, for example, the growth of droplets into raindrops in meteorology [52], the evolution of clouds of aerosol particles in atmospheric science [17], [23], the formation of large-scale structures in the universe [54], the formation of protostellar clusters within galaxies [3], [53], growth 
of dust in planet formation [15], population genetics [57], algal coagulation model [32]. In all these cases the real system only contains a finite, although very large, number of entities and it is implicitly assumed that the evolution of their number density is approximated in some sense by the solution of equation (1.1). Our main result means that the solution of this equation is actually an approximation, in strong $L^{1}$ sense, of the density function of a finite, but very large, set of coalescing entities, that follow a Marcus-Lushnikov process with bounded coalescing kernel. It may be difficult to establish whether the real finite system of coalescing entities that one is considering can be described by a Marcus-Lushnikov process, and this is an important aspect of modeling (see for example [2], [16], [43] where several cases are discussed). We also would like to mention that, using similar arguments, our main result may be extended to recover some coagulation-fragmentation equations as, for example, those in [1], [23], [44], [45], and [58].

As indicated before, the Marcus-Lushnikov process was introduced in [43] and later in [25], [41] and [56]. In these four references the authors obtain and study the evolution equation satisfied by the probability of the stochastic process, called joint frequency or mass distribution function. From that equation, they deduce the system of equations satisfied by the moments of the mass distribution function. The conditions under which this system may be approximated by the coagulation equation are discussed. In particular, it is seen that this depends on the absence of correlation between the numbers of particles of different mass (cf. also [52] for the continuous case).

In [22], [33], [34], [47] the authors consider the Marcus-Lushnikov process itself. The weak convergence of suitably rescaled versions of this process, called stochastic coalescents, towards the solutions of the coagulation equation is proved, under different conditions on the coagulation kernel $A$, both in the discrete and the continuous case. In [20] a different stochastic process is shown to approximate also the coagulation equation by using the equation for the mass density. A rate of convergence is obtained in [12]. The same process is used in [59] to study the so called gelation time that appears for some kind of coagulation kernels $A$. A combination of the Marcus-Lushnikov process with spatial inhomogeneity is considered in [30], [31] for bounded kernels, in [11] for sub linear kernels and in [60] for more general kernels. Rigorous derivations of spatially inhomogeneous coagulation equations from systems of diffusing spherical particles, interacting at contact, have been obtained in [48]. All these references on spatially inhomogeneous systems consider directly the stochastic particle process and prove its weak convergence in some suitable sense. In general, such probabilistic results can be interpreted in terms of law of large numbers.

Although the study of the stochastic process by probabilistic methods has proved to be very fruitful, we believe that the questions raised in [25], [41], [43] and [56], about the system of deterministic equations satisfied by the moments, are of interest. In particular, the study of the case of continuous masses via the equations for the mass distribution functions, applying techniques from classical kinetic theory is worthwhile, for itself and for further work [21]. That leads very naturally to a set of equations for the correlation functions $f_{j}$ (see (1.3)) that has been discussed to some extent in [25], [41], [43] and [56]. The introduction of the rescaled correlation functions $f_{j}^{V}$ (see (1.4)), leads to a system of equations (see (5.3) below) similar to the BBGKY hierarchy that appears in the study of many particles hamiltonian systems (see e.g. [49] and references therein). Notice that in such cases the underlying microscopic dynamics is deterministic (since it is given by the Newton equations associated to the Hamiltonian under consideration), while the dynamics of the coalescing particles is probabilistic, given by the Marcus-Lushnikov process. Nevertheless, the system of countably coupled equations obtained 
for the rescaled correlation functions $f_{j}^{V}$ may be treated with arguments similar to those that are used for the classical BBGKY hierarchy and our convergence result (1.8) may be stated in th! e same language, in terms of propagation of chaos.

Such arguments, inspired from many particles hamiltonian systems, have already been successfully used in [35], [36], [37] to derive a general class of multilinear Boltzmann-like integro-differential equations from finite systems of particles. Equation (1.1) is an example of bilinear model, that in the general form may be written as follows:

$$
\begin{array}{r}
\frac{\partial f}{\partial t}(t, m)=\frac{1}{2} \int_{0}^{\infty} \int_{0}^{\infty} B\left(m ; m_{1}, m_{2}\right) a\left(m_{1}, m_{2}\right) f\left(t, m_{1}\right) f\left(t, m_{2}\right) d m_{1} d m_{2}+ \\
-f(t, m) \int_{0}^{\infty} a\left(m, m_{1}\right) f\left(t, m_{1}\right) d m_{1},
\end{array}
$$

where $a\left(m_{1}, m_{2}\right)$ is an encounter rate, $B\left(m ; m_{1}, m_{2}\right)$ is a probability density and $B\left(m ; m_{1}, m_{2}\right) a\left(m_{1}, m_{2}\right)$ is a transition rate. It is proved in [35] and [36] that propagation of chaos holds for a suitable particle dynamics and that equation (1.9) is recovered in the limit where the number of particles $N$ tends to infinity. This result covers in particular the case of the coagulation equation (1.1) for a suitable choice of $a$ and $B$. The finite particle dynamics, built in [35] and [36] to approximate equation (1.9), is a Markov jump process, that may also be defined for more general bilinear [36] and multilinear [37] equations (see also [38]) but that is very different from the Marcus-Lushnikov process that we are considering here. Notice for example that the process used in [35], [36] conserves the number of particles, while in the Marcus-Lushnikov process that number decreases. Therefore, our results and those in [35], [36] concern different finite systems of particles.

On the other hand, as in [35] and [36], our method is based on the use of some analog of the classical BBGKY hierarchy for a suitable dynamics. Nevertheless, our choice of particles dynamics leads to the use of what we call correlation functions (by analogy with the statistical mechanics picture). By their physical meaning, their normalization and the compatibility rules that they satisfy, these functions are different from the $s$-individual marginal densities used in [35], [36].

Using similar arguments, propagation of chaos for spatially inhomogeneous systems of diffusive particles was proved in [39] for the constant kernel, but the proof does not work in the homogeneous case.

The arguments in [41] suggest that the validity of the propagation of chaos property for the Marcus-Lushnikov dynamics should depend on the coagulation kernel, and in particular on the occurrence or not of the gelation phenomena. On the other hand, the same arguments, and the rigorous results obtained in [22], [33], [34], [47], indicate that propagation of chaos should hold for non gelling kernels. The simplest case among non gelling kernels are the bounded kernels and this is exactly the class considered in our Theorem 1.1. It turns out that in that case the proof is simple and clear because the suitable functional space is easily seen to be globally preserved along the time evolution. The boundedness hypothesis imposed on the kernel $A$ is of course a restriction for the applications, but bounded kernels have been often considered in the mathematical literatu! re as a natural first step towards a better understanding of more general cases. We have not tried yet to extend our arguments to more general non gelling kernels. On the other hand, we believe that, very probably, different arguments are needed to study the propagation of chaos in coagulation systems with gelling kernels. 
Let us recall that, for mean-field models of classical particles with suitable two body potential, it has been proved that the propagation of chaos holds and, in the limit of an infinite number of particles, the Vlasov equation describes the time evolution of the particle density (see e.g. [10], [13], [46]). This result may be seen as a law of large numbers and its proof is based on the use of the so called empirical distribution. As explained in [27], it relies upon the two following facts. First, the empirical distribution is a weak solution of the Vlasov equation. Second, weak solutions to the Vlasov equation are continuous with respect to the initial datum in the topology of the weak convergence of the measures. No proof of this result based on the direct control of the BBGKY hierarchy is known (cf. for example the discussion in Section 1.4 of [49]).

The plan of the paper is the following. In Section 2 we briefly recall the description of the finite particle system and present an existence and uniqueness result for the system of equations satisfied by the mass distribution functions. In Section 3 we introduce the correlation functions and deduce the system of equations that they satisfy. Two simple time asymptotic properties of that system are derived in Section 4. The rescaled system is introduced in Section 5 where the main theorem is proved.

\section{The particle system}

We want to describe a finite system of particles, contained in a finite volume $V$ and whose number $N$ is not fixed through time. To this end we consider the probability space defined as follows.

The sample space is:

$$
\begin{aligned}
& \Omega=\bigcup_{N=1}^{\infty}\left(N, \mathscr{P}_{N}\left(\mathbb{R}^{+}\right)\right) \subset \mathbb{N}^{*} \times \mathscr{P}\left(\mathbb{R}^{+}\right), \\
& \mathscr{P}_{N}\left(\mathbb{R}^{+}\right): \text {subsets of } \mathbb{R}^{+} \text {with cardinal } N .
\end{aligned}
$$

It is the set of all the pairs $\left(N, \omega_{N}\right)$ where $N \in \mathbb{N}^{*}$ and $\omega_{N}$ is any finite subset of $N$ positive real numbers $m_{1}, m_{2}, \cdots, m_{N}$. For every time $t \geq 0$ the probability distribution of each state $\left(N,\left(m_{1}, \ldots, m_{N}\right)\right) \in \Omega$ is $P_{N}\left(t, m_{1}, \ldots, m_{N}\right) / N$ ! where $\left\{P_{N}(t)\right\}_{N \in \mathbb{N}^{*}}$ is a sequence of non negative functions $P_{N}(t)=P_{N}(t, \cdot)$, each of them defined on $\mathscr{P}_{N}\left(\mathbb{R}^{+}\right)$and normalized according to:

$$
\sum_{N=1}^{\infty} \frac{1}{N !} \int_{0}^{\infty} d m_{1} \cdots \int_{0}^{\infty} d m_{N} P_{N}\left(t, m_{1}, \cdots, m_{N}\right)=1 .
$$

The functions $P_{N}\left(t, m_{1}, \ldots, m_{N}\right)$ are assumed to be symmetric with respect to any permutation of the indices $1, \ldots, N$ and no restrictions are imposed on the range of mass values other than $m_{i}>0$ for $i=1, \ldots, N$. Each $P_{N}=P_{N}\left(t, m_{1}, \ldots, m_{N}\right)$ is the mass distribution function of the $N$-particle configuration $\left(m_{1}, \ldots, m_{N}\right)$. For every $N \in \mathbb{N}^{*}$ the function:

$$
P(t, N)=\frac{1}{N !} \int_{0}^{\infty} d m_{1} \cdots \int_{0}^{\infty} d m_{N} P_{N}\left(t, m_{1}, \cdots, m_{N}\right)
$$

is the probability that at time $t$ the system is constituted by $N$ particles and the normalization (2.3) is natural. 
Remark 2.1 The factor $1 / N$ ! in definition (2.4) is needed to compensate for counting all the $N$ ! physically equivalent ways of arranging the masses $m_{1}, \ldots, m_{N}$ in order of size.

Let $\mathcal{A}_{V}(m, \mu) \geq 0$ be the rate (per unit time) at which a single particle of mass $m$ collides and coalesces with a particle of mass $\mu$ (given only that each is in the considered spatial region of volume $V)$. We assume that:

$$
\mathcal{A}_{V}(m, \mu)=\mathcal{A}_{V}(\mu, m)
$$

as it is reasonable from a physical point of view.

In a finite coalescing system the number of particles will be varying along the time evolution (actually it will be decreasing) so what is meaningful is to consider the time evolution of the sequence of mass distribution functions $\left\{P_{N}(t)\right\}_{N \in \mathbb{N}^{*}}$. As explained in [25], [41], [43] and [56] the evolution equations for the mass distribution functions are:

$$
\begin{aligned}
\partial_{t} P_{N} & =\frac{1}{2} \sum_{\ell=1}^{N} \int_{0}^{m_{\ell}} d \mu \mathcal{A}_{V}\left(m_{\ell}-\mu, \mu\right) P_{N+1}\left(m_{1}, \ldots, m_{\ell-1}, m_{\ell}-\mu, m_{\ell+1}, \ldots, m_{N}, \mu, t\right)+ \\
& -\frac{1}{2} \sum_{\substack{\ell=1 \\
N}}^{N} \sum_{\substack{q=1 \\
q \neq \ell}}^{N} P_{N}\left(m_{1}, \ldots, m_{N}, t\right) \mathcal{A}_{V}\left(m_{\ell}, m_{q}\right), \quad N=1,2, \ldots
\end{aligned}
$$

The first term on the right hand side of (2.6) is the gain term and it describes the positive contribution due to the coagulation of a particle of mass $\mu$, with $\mu \leq m_{\ell}$, with a particle of mass $m_{\ell}-\mu$ (in an $N+1$-particle configuration), giving rise to a particle of mass $m_{\ell}$ (in an $N$-particle configuration). The second term on the right hand side of (2.6) is the loss term and it describes the negative contribution due to the coagulation of a particle of mass $m_{\ell}$ with a particle of mass $m_{q}$ (in an $N$-particle configuration), giving rise to a particle of mass $m_{\ell}+m_{q}$ (in an $N$-1-particle configuration). That set of equations completely neglects the contributions due, either to many-boby collisions (e.g., three-body collisions passing from an $N+2$ configuration to an $N$ configuration or from an $N$ configuration to an $N-2$ configuration ), or to the occurrence of multiple binary collisions (e.g., double binary collision again passing from an $N+2$ (or $N$ ) configuration to an $N$ (or $N-2$ ) configuration).

An important feature of system (2.6) is the conservation of the total mass. More precisely, denoting by $M_{N}$ the total mass of the configuration $m_{1}, \ldots, m_{N}$, i.e. $m_{1}+\cdots+m_{N}=M_{N}$, then the only processes that have a non zero contribution in the time variation of the distribution $P_{N}\left(m_{1}, \ldots, m_{N}, t\right)$ are those associated with configurations with the same total mass $M_{N}$. In fact, the gain term in (2.6) takes into account $N+1$-particle configurations with total mass $m_{1}+\cdots+m_{\ell}-\mu+\cdots+m_{N}+\mu=$ $M_{N}$ and the loss term in (2.6) leads to $N-1$ particle configurations in which the total mass is $m_{1}+\cdots+\left(m_{\ell}+m_{q}\right)+\cdots+m_{N}=M_{N}$.

The system of equations (2.6) has been studied in [25], [41], [43] and [56]. For the sake of completeness we present in this Section an existence and uniqueness result that suits our purposes. 


\subsection{The coalescence rate}

In general the coagulation kernel $\mathcal{A}_{V}\left(m_{1}, m_{2}\right)$ at which two particles of masses $m_{1}$ and $m_{2}$ coalesce in a volume $V$, has the following form:

$$
\mathcal{A}_{V}\left(m_{1}, m_{2}\right)=C_{V} \mathcal{A}\left(m_{1}, m_{2}\right) .
$$

The function $\mathcal{A}\left(m_{1}, m_{2}\right)$ encodes only the dependence of the coagulation rate on the masses $m_{1}$ and $m_{2}$. The term $C_{V}$ contains the dependence of that rate with respect to the volume $V$ as well as that on other physical properties of the coalescence process under consideration. An example (considered for instance in [25] and [43] ) is the following:

$$
C_{V}=\frac{1}{V} E\left(\left|v_{1}-v_{2}\right|\right) \pi
$$

where $E\left(\left|v_{1}-v_{2}\right|\right)$ is the average relative velocity of the two particles. The volume dependence of $C_{V}$ takes into account the fact that the coagulation rate increases as the proportion of the volume occupied by the particles with respect to the total volume $V$ increases. That gives a dependence inversely proportional to $V$, i.e. like $1 / V$. We consider in this paper the simplest possible case, namely:

$$
\begin{aligned}
& \mathcal{A}_{V}\left(m_{1}, m_{2}\right)=\frac{A\left(m_{1}, m_{2}\right)}{V}, \\
& 0 \leq A\left(m_{1}, m_{2}\right) \leq C_{A} .
\end{aligned}
$$

We assume in the sequel, without any loss of generality, that $C_{A}=1$, namely:

$$
\begin{aligned}
& \mathcal{A}_{V}\left(m_{1}, m_{2}\right)=\frac{A\left(m_{1}, m_{2}\right)}{V}, \\
& 0 \leq A\left(m_{1}, m_{2}\right) \leq 1
\end{aligned}
$$

Other kernels are considered in [21].

\section{$2.2 \quad$ The initial data}

We consider system (2.6) with initial datum:

$$
P_{N}^{0}\left(\mathbf{m}_{N}\right)=\left\{\begin{array}{l}
\left(N_{0}\right) ! f_{0}\left(m_{1}\right) \ldots f_{0}\left(m_{N_{0}}\right), \text { if } N=N_{0}, \\
0 \text { if } N \neq N_{0},
\end{array}\right.
$$

where the function $f_{0}$ is such that:

$$
f_{0}(m) \geq 0 \quad \text { a.e, } \quad \int_{0}^{\infty} d m f_{0}(m)=1 .
$$

Choosing the initial datum as in (2.13)-(2.14), and defining:

$$
P^{0}(N)=\frac{1}{N !} \int_{0}^{\infty} d m_{1} \ldots \int_{0}^{\infty} d m_{N} P_{N}^{0}\left(m_{1}, \ldots, m_{N}\right)
$$


for every $N \in \mathbb{N}^{*}$, we obtain

$$
\sum_{N=1}^{\infty} P^{0}(N)=1
$$

and condition (2.3) is satisfied at time $t=0$. With that choice of initial datum we have:

$$
P^{0}(N)=\delta\left(N-N_{0}\right),
$$

i.e. at $t=0$ our system has exactly $N_{0}$ particles.

\subsection{Well-posedeness of the equation for $P_{N}(t)$ for bounded kernel}

For every $N \in \mathbb{N}^{*}$ we define the operator $G_{N}$, mapping $N+1$-particle functions into $N$-particle functions, as follows:

$$
\begin{aligned}
G_{N}\left[Q_{N+1}\right]\left(\mathbf{m}_{N}\right)= & \frac{1}{2 V} \sum_{\ell=1}^{N} \int_{0}^{m_{\ell}} d m_{N+1} A\left(m_{\ell}-m_{N+1}, m_{N+1}\right) \times \\
& \times Q_{N+1}\left(m_{1}, \ldots, m_{\ell-1}, m_{\ell}-m_{N+1}, m_{\ell+1}, \ldots, m_{N}, m_{N+1}\right),
\end{aligned}
$$

where $Q_{N+1} \in L^{1}\left(\left(\mathbb{R}^{+}\right)^{N+1}\right)$, and we denote:

$$
\mathbf{m}_{N}:=\left(m_{1}, \ldots, m_{N}\right) .
$$

We shall use the following notation:

$$
\int d \mathbf{t}_{N}:=\int_{0}^{t} d t_{1} \ldots \int_{0}^{t_{N-1}} d t_{N}
$$

We also introduce the following set:

$$
\mathcal{H}=\left\{\left\{f_{N}\right\}_{N \in \mathbb{N}^{*}} ; f_{k} \in L^{1}\left(\left(\mathbb{R}^{+}\right)^{k}\right), k \in \mathbb{N}^{*}\right\} .
$$

Definition 2.2 We say that a sequence $\left\{P_{N}(t)\right\}_{N \in \mathbb{N}^{*}} \in \mathcal{H}$ solves the system (2.6) if, for every $t>0$ and every $N \in \mathbb{N}^{*}$, each term in (2.6) belongs to $L^{1}\left(\left(\mathbb{R}^{+}\right)^{N}\right)$ and the equality holds in $L^{1}\left(\left(\mathbb{R}^{+}\right)^{N}\right)$.

Lemma 2.3 For every $Q_{N+1} \in L^{1}\left(\left(\mathbb{R}^{+}\right)^{N+1}\right)$ :

$$
\begin{aligned}
&\left\|G_{N}\left[Q_{N+1}\right]\right\|_{L^{1}\left(\left(\mathbb{R}^{+}\right)^{N}\right)} \leq \frac{N}{2 V}\left\|Q_{N+1}\right\|_{L^{1}\left(\left(\mathbb{R}^{+}\right)^{N+1}\right)} . \\
& \text { If } A \equiv 1, Q_{N+1} \in L^{1}\left(\left(\mathbb{R}^{+}\right)^{N+1}\right) \text { and } Q_{N+1} \geq 0 \text { then } \\
&\left\|G_{N}\left[Q_{N+1}\right]\right\|_{L^{1}\left(\left(\mathbb{R}^{+}\right)^{N}\right)}=\frac{N}{2 V}\left\|Q_{N+1}\right\|_{L^{1}\left(\left(\mathbb{R}^{+}\right)^{N+1}\right)} .
\end{aligned}
$$


Proof of Lemma 2.3 We start proving (2.21). To this end we write:

$$
\begin{aligned}
& \|\left. G_{N}\left[Q_{N+1}\right]\right|_{L^{1}\left(\left(\mathbb{R}^{+}\right)^{N}\right)}=\int_{0}^{\infty} d m_{1} \cdots \int_{0}^{\infty} d m_{N}\left|G_{N}\left[Q_{N+1}\right]\left(m_{1}, \cdots, m_{N}\right)\right| \\
& \leq \frac{1}{2 V} \sum_{\ell=1}^{N} \int_{0}^{\infty} d m_{1} \cdots \int_{0}^{\infty} d m_{N} \int_{0}^{m_{\ell}} d m_{N+1}\left|Q_{N+1}\left(m_{1}, m_{2}, \cdots, m_{\ell}-m_{N+1}, \cdots, m_{N+1}\right)\right| \\
& =\frac{1}{2 V} \sum_{\ell=1}^{N} \underbrace{\int_{0}^{\infty} d m_{1} \cdots \int_{0}^{\infty} d m_{N}}_{m_{i} \neq m_{\ell}} \int_{0}^{\infty} d m_{\ell} \int_{0}^{m_{\ell}} d m_{N+1}\left|Q_{N+1}\left(m_{1}, m_{2}, \cdots, m_{\ell}-m_{N+1}, \cdots, m_{N+1}\right)\right|,
\end{aligned}
$$

where we have used (2.12) to get the first inequality and, in the last step, we have used that the domains of integration of the variables $m_{i}$ for $i=1, \cdots, N$ are independent and therefore the integrations can be performed in arbitrary order. The final and trivial step is to use Fubini's theorem in the integrals with respect to $m_{\ell}$ and $m_{N+1}$. That may be done since, by hypothesis, $Q_{N+1} \in L^{1}\left(\left(\mathbb{R}^{+}\right)^{N+1}\right)$ and therefore, for almost every $\left(m_{1}, m_{2}, \cdots, m_{\ell-1}, m_{\ell+1}, \cdots, m_{N}\right), Q_{N+1}\left(m_{1}, m_{2}, \cdots, m_{\ell}, \cdots, m_{N}, m_{N+1}\right) \in$ $L^{1}\left(\left(\mathbb{R}^{+}\right)^{2}\right)$. If we also have $A \equiv 1$ and $Q_{N+1} \geq 0$, the inequality in $(2.23)$ is then an equality and we deduce $(2.22)$.

We can state now the existence and uniqueness result of solutions for system (2.6).

Theorem 2.4 Suppose that $\mathcal{A}_{V} \leq V^{-1}$ according to (2.11), (2.12). Then, the Cauchy problem for the system (2.6) with initial data $\left\{P_{N}^{0}\right\}_{N \in \mathbb{N}^{*}}$ defined in (2.13)-(2.14) has a solution $\left\{P_{N}(t)\right\}_{N \in \mathbb{N}^{*}} \in \mathcal{H}$ for any $t$ and such that $P_{N} \in \mathbf{C}^{\infty}\left([0,+\infty), L^{1}\left(\left(\mathbb{R}^{+}\right)^{N}\right)\right)$ for every $N \in \mathbb{N}^{*}$. Such a solution is given by

$$
P_{N}\left(t, \mathbf{m}_{N}\right)= \begin{cases}\int d \mathbf{t}_{N_{0}-N} e^{-\frac{S\left(m_{N}\right)}{2 V}\left(t-t_{1}\right)} G_{N}\left[\ldots \left[e^{-\frac{S\left(m_{N_{0}-1}\right)}{2 V}\left(t_{N_{0}-N-1}-t_{N_{0}-N}\right)} G_{N_{0}-1}[\right.\right. \\ & e^{\left.\left.\left.-\frac{S\left(m_{N_{0}}\right)}{2 V} t_{N_{0}-N} P_{N_{0}}^{0}\left(\mathbf{m}_{N_{0}}\right)\right]\right] \ldots\right],} \text { for } 1 \leq N<N_{0}, \\ e^{-\frac{S\left(m_{N_{0}}\right)}{2 V} t} P_{N_{0}}^{0}\left(\mathbf{m}_{N_{0}}\right), & \text { for } N=N_{0}, \\ 0, & \text { for } N>N_{0},\end{cases}
$$

where

$$
S\left(\boldsymbol{m}_{N}\right)=\sum_{\ell=1}^{N} \sum_{\substack{q=1 \\ q \neq \ell}}^{N} A\left(m_{\ell}, m_{q}\right) .
$$

Moreover, $P_{N}(t) \geq 0$ for every $t>0$ and:

$$
\sum_{N=1}^{\infty} P(t, N)=1,
$$

with $\quad P(t, N):=\frac{1}{N !} \int_{0}^{\infty} d m_{1} \cdots \int_{0}^{\infty} d m_{N} P_{N}\left(t, m_{1}, \cdots, m_{N}\right)$. 
That solution is unique in the set of sequences $\left\{\bar{P}_{N}(t)\right\}_{N \in \mathbb{N}^{*}} \in \mathcal{H}$ such that $\bar{P}_{N} \in \mathbf{C}^{1}\left([0,+\infty) ; L^{1}\left(\left(\mathbb{R}^{+}\right)^{N}\right)\right), \bar{P}_{N} \geq 0$ for all $N \in \mathbb{N}^{*}$ and the function $h_{\bar{P}}(t):=\sum_{N=1}^{\infty} \bar{P}(t, N)$ satisfies $h_{\bar{P}} \in \mathbf{C}^{1}([0,+\infty))$.

Proof of Theorem 2.4 By our hypothesis we have $P_{N_{0}}^{0} \in L^{1}\left(\left(\mathbb{R}^{+}\right)^{N_{0}}\right)$. By Lemma 2.3 we deduce that, for every $1 \leq N \leq N_{0}$ :

$$
G_{N} G_{N+1} \ldots G_{N_{0}-1}\left[P_{N_{0}}^{0}\right] \in L^{1}\left(\left(\mathbb{R}^{+}\right)^{N}\right) .
$$

Then, for any $a$ and $\tau$, the function

$$
\eta\left(t, \mathbf{m}_{N}\right)=e^{a(t-\tau)} G_{N} G_{N+1} \ldots G_{N_{0}-1}\left[P_{N_{0}}^{0}\right]\left(\mathbf{m}_{N}\right)
$$

satisfies:

$$
\eta \in \mathbf{C}^{\infty}\left([0,+\infty), L^{1}\left(\left(\mathbb{R}^{+}\right)^{N}\right)\right) .
$$

It then follows that $P_{N}(t)$, given by $(2.24)$, is such that $P_{N} \in \mathbf{C}^{\infty}\left([0,+\infty), L^{1}\left(\left(\mathbb{R}^{+}\right)^{N}\right)\right)$.

We check now that the above sequence $\left\{P_{N}(t)\right\}_{N \in \mathbb{N}^{*}}$ satisfies system (2.6). The equations for $N \geq N_{0}+1$ are trivially satisfied. Suppose now that $N \leq N_{0}-1$. Taking the time derivative of the left hand side of (2.24) we obtain:

$$
\begin{aligned}
& \frac{\partial P_{N}}{\partial t}\left(t, \mathbf{m}_{N}\right)=-\frac{S\left(\mathbf{m}_{N}\right)}{2 V} P_{N}\left(t, \mathbf{m}_{N}\right)+ \\
&+\int_{0}^{t} d t_{2} \int_{0}^{t_{2}} d t_{3} \ldots \int_{0}^{t_{N_{0}-N-1}} d t_{N_{0}-N} G_{N}\left[e^{-\frac{S\left(\mathbf{m}_{N+1}\right)}{2 V}\left(t-t_{2}\right)} G_{N+1}[\ldots\right. \\
&\left.\left.\quad \ldots G_{N_{0}-2}\left[e^{-\frac{S\left(\mathbf{m}_{\left.N_{0}-1\right)}\right)}{2 V} t_{N_{0}-N-1} G_{N_{0}-1}}\left[e^{-\frac{S\left(\mathbf{m}_{N_{0}}\right)}{2 V} t_{N_{0}-N}} P_{N_{0}}^{0}\left(\mathbf{m}_{N_{0}}\right)\right]\right] \ldots\right]\right] \\
&=-\frac{S\left(\mathbf{m}_{N}\right)}{2 V} P_{N}\left(t, \mathbf{m}_{N}\right)+ \\
&+G_{N}\left[\int_{0}^{t} d t_{1} \int_{0}^{t_{1}} d t_{2} \ldots \int_{0}^{t_{N_{0}-N-2}} d t_{N_{0}-N-1} e^{-\frac{S\left(\mathbf{m}_{N+1}\right)}{2 V}\left(t-t_{1}\right)} G_{N+1}[\ldots\right. \\
& \ldots\left[e ^ { - \frac { S ( \mathbf { m } _ { N _ { 0 } - 1 ) } } { 2 V } t _ { N _ { 0 } - N - 2 } G _ { N _ { 0 } - 1 } } \left[e^{\left.\left.\left.-\frac{S\left(\mathbf{m}_{N_{0}}\right)}{2 V} t_{N_{0}-N-1} P_{N_{0}}^{0}\left(\mathbf{m}_{N_{0}}\right)\right]\right] \ldots\right] .}\right.\right.
\end{aligned}
$$

Since

$$
\begin{aligned}
& \int_{0}^{t} d t_{1} \int_{0}^{t_{1}} d t_{2} \ldots \int_{0}^{t_{N_{0}-N-2}} d t_{N_{0}-N-1} e^{-\frac{S\left(\mathbf{m}_{N+1}\right)}{2 V}\left(t-t_{1}\right)} G_{N+1}[\ldots \\
& \left.\ldots\left[e^{-\frac{S\left(\mathbf{m}_{N_{0}-1}\right)}{2 V} t_{N_{0}-N-2} G_{N_{0}-1}}\left[e^{-\frac{S\left(\mathbf{m}_{N_{0}}\right)}{2 V} t_{N_{0}-N-1}} P_{N_{0}}^{0}\left(\mathbf{m}_{N_{0}}\right)\right]\right] \ldots\right] \equiv P_{N+1}\left(t, \mathbf{m}_{N+1}\right),
\end{aligned}
$$

we deduce that $P_{N}$ satisfies the equation

$$
\frac{\partial P_{N}}{\partial t}\left(t, \mathbf{m}_{N}\right)=-\frac{S\left(\mathbf{m}_{N}\right)}{2 V} P_{N}\left(t, \mathbf{m}_{N}\right)+G_{N}\left[P_{N+1}\right]\left(t, \mathbf{m}_{N}\right),
$$


that is exactly the $N^{\text {th }}$ equation of system (2.6).

If $N=N_{0}$, we have:

$$
\begin{aligned}
\frac{\partial P_{N_{0}}}{\partial t}\left(t, \mathbf{m}_{N_{0}}\right) & =-\frac{S\left(\mathbf{m}_{N_{0}}\right)}{2 V} P_{N}\left(t, \mathbf{m}_{N_{0}}\right) \\
& \equiv-\frac{S\left(\mathbf{m}_{N_{0}}\right)}{2 V} P_{N}\left(t, \mathbf{m}_{N_{0}}\right)+G_{N_{0}}\left[P_{N_{0}+1}\right]\left(t, \mathbf{m}_{N_{0}}\right)
\end{aligned}
$$

since, by $(2.24), P_{N_{0}+1}(t) \equiv 0$ for any $t$.

We check now (2.26). To this end we first notice that, by $(2.24)$ :

$$
\sum_{N=1}^{\infty} P(t, N)=\sum_{N=1}^{N_{0}} \frac{1}{N !} \int_{0}^{\infty} d m_{1} \ldots \int_{0}^{\infty} d m_{N} P_{N}\left(t, m_{1}, \ldots, m_{N}\right) .
$$

Therefore, by (2.6) and (2.17), we find

$$
\begin{aligned}
\frac{d}{d t} \sum_{N=1}^{\infty} P(t, N)= & -\sum_{N=1}^{N_{0}} \frac{N(N-1)}{N ! 2 V} \int d \mathbf{m}_{N} A\left(m_{1}, m_{2}\right) P_{N}\left(\mathbf{m}_{N}, t\right)+\sum_{N=1}^{N_{0}} \frac{1}{N !} \int d \mathbf{m}_{N} G_{N}\left[P_{N+1}\right](t) \\
= & -\sum_{N=1}^{N_{0}} \frac{N(N-1)}{N ! 2 V} \int d \mathbf{m}_{N} A\left(m_{1}, m_{2}\right) P_{N}\left(\mathbf{m}_{N}, t\right)+ \\
& +\sum_{N=1}^{N_{0}-1} \frac{N}{N ! 2 V} \int d \mathbf{m}_{N+1} A\left(m_{1}, m_{2}\right) P_{N+1}\left(\mathbf{m}_{N+1}, t\right) \\
= & -\sum_{N=1}^{N_{0}} \frac{N(N-1)}{N ! 2 V} \int d \mathbf{m}_{N} A\left(m_{1}, m_{2}\right) P_{N}\left(\mathbf{m}_{N}, t\right)+ \\
& +\sum_{M=2}^{N_{0}} \frac{M-1}{(M-1) ! 2 V} \int d \mathbf{m}_{M} A\left(m_{1}, m_{2}\right) P_{M}\left(\mathbf{m}_{M}, t\right) \equiv 0 .
\end{aligned}
$$

Identity (2.26) follows from the fact that, by continuity and (2.16), one has:

$$
\sum_{N=1}^{N_{0}} P(t, N)=\sum_{N=1}^{N_{0}} P^{0}(N)=1
$$

In order to prove the uniqueness, we first show that for any solution $\left\{\bar{P}_{N}(t)\right\}_{N \in \mathbb{N}^{*}}$, satisfying $\bar{P}_{N}(t) \geq 0$ and such that the auxiliary function $h_{\bar{P}}(t) \equiv \sum_{N=1}^{\infty} \bar{P}(t, N)$ satisfies $h_{\bar{P}} \in C^{1}((0,+\infty))$, 
we have $\bar{P}_{N}(t) \equiv 0$ for all $t$ and $N \geq N_{0}+1$. To this end we write:

$$
\begin{aligned}
\frac{d}{d t} \sum_{N=N_{0}+1}^{\infty} \bar{P}(t, N)= & -\sum_{N=N_{0}+1}^{\infty} \frac{N(N-1)}{N ! 2 V} \int d \mathbf{m}_{N} A\left(m_{1}, m_{2}\right) \bar{P}_{N}\left(\mathbf{m}_{N}, t\right)+ \\
& +\sum_{N=N_{0}+1}^{\infty} \frac{1}{N !} \int d \mathbf{m}_{N} G_{N}\left[\bar{P}_{N+1}\right](t) \\
= & -\sum_{N=N_{0}+1}^{\infty} \frac{N(N-1)}{N ! 2 V} \int d \mathbf{m}_{N} A\left(m_{1}, m_{2}\right) \bar{P}_{N}\left(\mathbf{m}_{N}, t\right)+ \\
& +\sum_{N=N_{0}+1}^{\infty} \frac{N}{N ! 2 V} \int d \mathbf{m}_{N+1} A\left(m_{1}, m_{2}\right) \bar{P}_{N+1}\left(\mathbf{m}_{N+1}, t\right) \\
=- & \sum_{N=N_{0}+1}^{\infty} \frac{N(N-1)}{N ! 2 V} \int d \mathbf{m}_{N} A\left(m_{1}, m_{2}\right) \bar{P}_{N}\left(\mathbf{m}_{N}, t\right)+ \\
& +\sum_{N=N_{0}+2}^{\infty} \frac{N(N-1)}{N ! 2 V} \int d \mathbf{m}_{N} A\left(m_{1}, m_{2}\right) \bar{P}_{N}\left(\mathbf{m}_{N}, t\right) \\
= & -\frac{N_{0}\left(N_{0}+1\right)}{\left(N_{0}+1\right) ! 2 V} \int d \mathbf{m}_{N_{0}+1} A\left(m_{1}, m_{2}\right) \bar{P}_{N_{0}+1}\left(\mathbf{m}_{N_{0}+1}, t\right)<0 .
\end{aligned}
$$

Therefore:

$$
\sum_{N=N_{0}+1}^{\infty} \bar{P}(t, N) \leq \sum_{N=N_{0}+1}^{\infty} \bar{P}(0, N)=\sum_{N=N_{0}+1}^{\infty} P^{0}(N) .
$$

Since, by hypothesis, the right hand side of $(2.28)$ is zero and $\bar{P}_{N}(t) \geq 0$, we deduce that $\bar{P}_{N}(t) \equiv 0$ for every $N \geq N_{0}+1$ and $t \geq 0$. That leaves only a finite number of equations in the system (2.6), for $N=1, \cdots, N_{0}$. Moreover the equation for $N=N_{0}$ yields:

$$
P_{N_{0}}\left(t, \mathbf{m}_{N_{0}}\right)=P_{N_{0}}^{0}\left(\mathbf{m}_{N_{0}}\right) e^{-\frac{1}{2 V} S\left(\mathbf{m}_{N_{0}}\right) t} .
$$

Then, the finite system decouples and may be explicitly solved for $N=1, \cdots, N_{0}-1$ to obtain expression (2.24).

Remark 2.5 One would expect that for every initial data $\left\{P_{N}^{0}\right\}_{N \in \mathbb{N}^{*}} \in \mathcal{H}$ such that $P_{N}^{0} \geq 0$ for every $N$ and $\sum P^{0}(N)=1$, there exists a solution $\left\{P_{N}(t)\right\}_{N \in \mathbb{N}^{*}}$ such that, for every $N: P_{N}(t) \geq 0$, $P_{N} \in \mathbf{C}\left([0,+\infty) ; L^{1}\left(\left(\mathbb{R}^{+}\right)^{N}\right)\right) \cap \mathbf{C}^{1}\left((0,+\infty) ; L^{1}\left(\left(\mathbb{R}^{+}\right)^{N}\right)\right), h_{P} \in \mathbf{C}^{1}(0,+\infty)$ and $\sum_{N=1}^{\infty} P(t, N)=1$. Since such a result is not necessary for our main purpose we only prove the simpler result in Theorem 2.4. 


\section{Correlation Functions}

For any fixed $j \in \mathbb{N}^{*}$, we define the $j$-particle correlation function $f_{j}\left(m_{1}, \ldots, m_{j}, t\right)$ at time $t$ as

$$
f_{j}\left(m_{1}, \ldots, m_{j}, t\right)=\sum_{N=j}^{\infty} \frac{1}{(N-j) !} \int_{0}^{\infty} d m_{j+1} \ldots \int_{0}^{\infty} d m_{N} P_{N}\left(m_{1}, \ldots, m_{N}, t\right) .
$$

Some general properties of such functions $f_{j}$ are the following. At any time $t$ the expected (or mean) number of particles $\bar{N}(t)$ defined as

$$
\overline{N(t)}=\sum_{N=1}^{\infty} N P(t, N)
$$

satisfies

$$
\overline{N(t)}=\int_{0}^{\infty} f_{1}\left(m_{1}, t\right) d m_{1} .
$$

The function $f_{1}$ is then the density function associated to the average number of particles. More generally one may also define:

$$
\overline{(N(t))(N(t)-1) \ldots(N(t)-j+1)}=\sum_{N=1}^{\infty} N(N-1) \ldots(N-j+1) P(t, N),
$$

and then:

$$
\overline{(N(t))(N(t)-1) \ldots(N(t)-j+1)}=\left\|f_{j}\right\|_{L^{1}\left(\left(\mathbb{R}^{+}\right)^{j}\right)} .
$$

The functions $f_{j}$ 's will be called correlation functions since they satisfy properties (3.2), (3.3) and their definition is very similar to that of the classical correlation functions in statistical mechanics (see e.g. [50]).

As an immediate Corollary of Theorem 2.4 we have the following.

Corollary 3.1 Consider the solution $\left\{P_{N}(t)\right\}_{N \in \mathbb{N}^{*}}$ of the Cauchy problem (2.6)-(2.13)-(2.14), whose existence and uniqueness have been proved in Theorem 2.4. Then, for every $j \in \mathbb{N}^{*}$, the functions $f_{j}(t)$ defined as

$$
f_{j}\left(m_{1} \cdots, m_{j}, t\right)=\sum_{N=j}^{\infty} \frac{1}{(N-j) !} \int_{0}^{\infty} d m_{j+1} \cdots \int_{0}^{\infty} d m_{N} P_{N}\left(t, m_{1}, \ldots, m_{N}\right),
$$

satisfy:

(i) $\quad f_{j}(0)=\frac{\left(N_{0}\right) !}{\left(N_{0}-j\right) !} f_{0}^{\otimes j}, \forall j \in\left\{1, \ldots, N_{0}\right\} ; \quad f_{j}(0) \equiv 0, \forall j \geq N_{0}+1$,

(ii) $f_{j}(t) \geq 0 \forall j \geq 1$ and $\forall t \geq 0 ; \quad f_{j}(t) \equiv 0 \forall t \geq 0$ if $j \geq N_{0}+1$,

(iii) $f_{j} \in \mathbf{C}^{\infty}\left([0,+\infty) ; L^{1}\left(\left(\mathbb{R}^{+}\right)^{j}\right)\right)$,

(iv) $\left\|f_{j}(t)\right\|_{L^{1}\left(\left(\mathbb{R}^{+}\right)^{j}\right)} \leq 2^{j-1} N_{0} !\left(\frac{t}{V}\right)^{N_{0}-j} \sum_{N=0}^{N_{0}-j} \frac{2^{N}}{N !} t^{-N} V^{N}$ for $j=1, \cdots, N_{0}$. 
Proof of Corollary 3.1 Only (3.8) needs an explanation. It follows from the fact that, by definition:

$$
\left\|f_{j}(t)\right\|_{L^{1}\left(\left(\mathbb{R}^{+}\right)^{j}\right)}=\sum_{N=j+1}^{N_{0}} \frac{1}{(N-j) !}\left\|P_{N}(t)\right\|_{L^{1}\left(\left(\mathbb{R}^{+}\right)^{N}\right)},
$$

where we used that $P_{N}(t) \equiv 0$ for $N \geq N_{0}+1$. On the other hand, for every $N \leq N_{0}$ we have, by Theorem 2.4:

$$
\left\|P_{N}(t)\right\|_{L^{1}\left(\left(\mathbb{R}^{+}\right)^{N}\right)}=\int d \mathbf{t}_{N_{0}-N} \int d \mathbf{m}_{N} e^{-\frac{S\left(\mathbf{m}_{N}\right)}{2 V}\left(t-t_{1}\right)} G_{N} \ldots G_{N_{0}-1} e^{-\frac{S\left(\mathbf{m}_{N_{0}}\right)}{2 V} t_{N_{0}-N}} P_{N_{0}}\left(\mathbf{m}_{N_{0}}, 0\right) .
$$

Using iteratively Lemma 2.3 we deduce:

$$
\begin{aligned}
\left\|P_{N}(t)\right\|_{L^{1}\left(\left(\mathbb{R}^{+}\right)^{N}\right)} & \leq \frac{t^{N_{0}-N}}{\left(N_{0}-N\right) !} 2^{-\left(N_{0}-N\right)} V^{-\left(N_{0}-N\right)} N(N+1) \ldots\left(N_{0}-1\right)\left\|P_{N_{0}}(0)\right\|_{L^{1}\left(\left(\mathbb{R}^{+}\right)^{N_{0}}\right)} \\
& =\frac{t^{N_{0}-N}}{\left(N_{0}-N\right) !} 2^{-\left(N_{0}-N\right)} V^{-\left(N_{0}-N\right)} \frac{\left(N_{0}-1\right) !}{(N-1) !}\left\|P_{N_{0}}(0)\right\|_{L^{1}\left(\left(\mathbb{R}^{+}\right)^{N_{0}}\right)} .
\end{aligned}
$$

From (3.10), using assumptions (2.13)-(2.14) on the initial datum $P_{N_{0}}(0)$ and the inequality

$$
\forall a \geq b \geq 1: \quad \frac{(a-1) !}{(a-b) !(b-1) !} \leq 2^{a-1},
$$

we deduce:

$$
\begin{aligned}
\left\|P_{N}(t)\right\|_{L^{1}\left(\left(\mathbb{R}^{+}\right)^{N}\right)} & \leq \frac{t^{N_{0}-N}}{\left(N_{0}-N\right) !} 2^{-\left(N_{0}-N\right)} V^{-\left(N_{0}-N\right)} 2^{N_{0}-1}\left(N_{0}-N\right) !\left\|P_{N_{0}}(0)\right\|_{L^{1}\left(\left(\mathbb{R}^{+}\right)^{N_{0}}\right)} \\
& =2^{N-1} t^{N_{0}-N} V^{-\left(N_{0}-N\right)} N_{0} ! .
\end{aligned}
$$

Estimate (3.8) follows from (3.9) and (3.12).

\subsection{The system of equations for the functions $f_{j}$}

An immediate consequence of Corollary (3.1) is the following:

Theorem 3.2 Suppose that $\left\{P_{N}(t)\right\}_{N \in \mathbb{N}^{*}}$ is the unique solution of (2.6) with initial data $\left\{P_{N}^{0}\right\}_{N \in \mathbb{N}^{*}}$ defined in (2.13)-(2.14) and coagulation kernel $\mathcal{A}_{V} \leq V^{-1}$ according to (2.11), (2.12). Then the set of functions $\left\{f_{j}(t)\right\}_{j \in \mathbb{N}^{*}}$ defined in (3.4) solves the following problem:

$$
\begin{aligned}
\partial_{t} f_{j} & =\frac{1}{2 V} \sum_{\ell=1}^{j} \int_{0}^{m_{\ell}} d \mu A\left(m_{\ell}-\mu, \mu\right) f_{j+1}\left(m_{1}, \ldots, m_{\ell}-\mu, \ldots, m_{j}, \mu, t\right)+ \\
& -\frac{S\left(\mathbf{m}_{j}\right)}{2 V} f_{j}\left(m_{1}, \ldots, m_{j}, t\right)-\frac{1}{V} \sum_{\ell=1}^{j} \int_{0}^{\infty} d \mu A\left(m_{\ell}, \mu\right) f_{j+1}\left(m_{1}, \ldots, m_{j}, \mu, t\right), \quad j \in \mathbb{N}^{*} .
\end{aligned}
$$

Moreover the sequence $\left\{f_{j}(t)\right\}_{j \in \mathbb{N}^{*}}$ is the unique solution in $\mathbf{C}\left([0,+\infty) ; L^{1}\left(\left(\mathbb{R}^{+}\right)^{j}\right)\right) \cap$ $\mathbf{C}^{1}\left((0,+\infty) ; L^{1}\left(\left(\mathbb{R}^{+}\right)^{j}\right)\right)$ of (3.13) with initial data given as in (3.5). 
Proof of Theorem 3.2 Using the definition (3.4), a straightforward computation from (2.6) shows that for any $j \leq N_{0}$ the function $f_{j}$ satisfies:

$$
\begin{aligned}
\partial_{t} f_{j}\left(\mathbf{m}_{j}, t\right)= & \frac{1}{2 V} \sum_{N=j}^{N_{0}} \frac{1}{(N-j) !} \sum_{\ell=1}^{N} \int d \mathbf{m}_{N, j} \int_{0}^{m_{\ell}} d m_{N+1} A\left(m_{\ell}-m_{N+1}, m_{N+1}\right) \times \\
& \times P_{N+1}\left(m_{1}, \ldots, m_{\ell}-m_{N+1}, \ldots, m_{N+1}, t\right)+ \\
& -\sum_{N=j}^{N_{0}} \frac{1}{(N-j) !} \frac{1}{2 V} \sum_{\substack{\ell=1 \\
N}}^{N} \int d \mathbf{m}_{N, j} A\left(m_{\ell}, m_{q}\right) P_{N}\left(m_{1}, \ldots, m_{N}, t\right),
\end{aligned}
$$

where from now on we use the notation:

$$
\mathbf{m}_{N, j}:=\left(m_{j+1}, \ldots, m_{N}\right) \text { and } \int d \mathbf{m}_{N, j}:=\int_{0}^{\infty} d m_{j+1} \ldots \int_{0}^{\infty} d m_{N} .
$$

The first term in the right hand side of (3.14), or gain term, gives the following contributions:

$$
\begin{aligned}
& \frac{1}{2 V} \sum_{N=j}^{N_{0}} \frac{1}{(N-j) !} \sum_{\ell=1}^{N} \int d \mathbf{m}_{N, j} \int_{0}^{m_{\ell}} d m_{N+1} A\left(m_{\ell}-m_{N+1}, m_{N+1}\right) \times \\
& \quad \times P_{N+1}\left(m_{1}, \ldots, m_{\ell}-m_{N+1}, \ldots, m_{N+1}, t\right) \\
& =\frac{1}{2 V} \sum_{\ell=1}^{j} \int_{0}^{m_{\ell}} d m_{N+1} A\left(m_{\ell}-m_{N+1}, m_{N+1}\right) f_{j+1}\left(m_{1}, \ldots, m_{\ell}-m_{N+1}, \ldots, m_{j}, m_{N+1}, t\right)+ \\
& +\frac{1}{2 V} \sum_{N=j}^{N_{0}} \frac{N-j}{(N-j) !} \int d \mathbf{m}_{N, j+1} \int_{0}^{\infty} d m_{j+1} \int_{0}^{m_{j+1}} d m_{N+1} A\left(m_{j+1}-m_{N+1}, m_{N+1}\right) \times \\
& \quad \times P_{N+1}\left(\mathbf{m}_{j}, m_{j+1}-m_{N+1}, \ldots, m_{N+1}, t\right),
\end{aligned}
$$

where we have divided the sum with respect to $\ell$ in two parts, $1 \leq \ell \leq j$ and $j+1 \leq \ell \leq N$, and we have used the symmetry of $P_{N}$ with respect to any permutation of the indeces (that is preserved by the dynamics), to write:

$$
\begin{aligned}
& \sum_{\ell=j+1}^{N} \int_{0}^{m_{\ell}} d m_{N+1} A\left(m_{\ell}-m_{N+1}, m_{N+1}\right) P_{N+1}\left(m_{1}, \ldots, m_{\ell}-m_{N+1}, \ldots, m_{N+1}, t\right) \\
& =(N-j) \int_{0}^{m_{j+1}} d m_{N+1} A\left(m_{j+1}-m_{N+1}, m_{N+1}\right) P_{N+1}\left(m_{1}, \ldots, m_{j+1}-m_{N+1}, \ldots, m_{N+1}, t\right) .
\end{aligned}
$$

Using Fubini's theorem and definition (3.4) we obtain

$$
\begin{array}{r}
\frac{1}{2 V} \sum_{N=j}^{N_{0}} \frac{N-j}{(N-j) !} \int d \mathbf{m}_{N, j+1} \int_{0}^{\infty} d m_{j+1} \\
\int_{0}^{m_{j+1}} d m_{N+1} A\left(m_{j+1}-m_{N+1}, m_{N+1}\right) \times \\
\quad \times P_{N+1}\left(\mathbf{m}_{j}, m_{j+1}-m_{N+1}, \ldots, m_{N+1}, t\right) \\
=\frac{1}{2 V} \int_{0}^{\infty} d m_{N+1} \int_{0}^{\infty} d m_{j+1} A\left(m_{j+1}, m_{N+1}\right) f_{j+2}\left(m_{1}, \ldots, m_{j+1}, m_{N+1}, t\right) .
\end{array}
$$


On the other hand, by simple algebraic manipulations, the second term in the right hand side of (3.14), or loss term, gives:

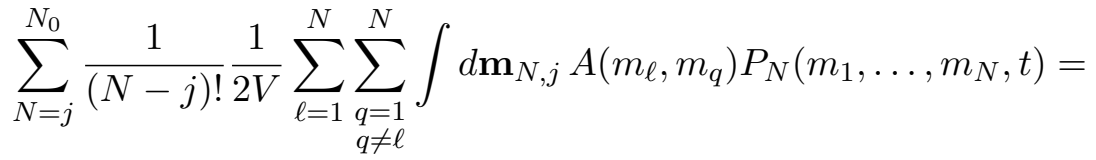

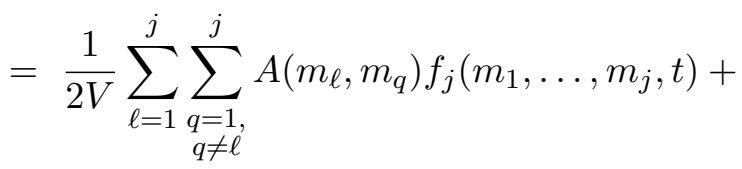

$$
\begin{aligned}
& +\frac{1}{V} \sum_{\ell=1}^{j} \int_{0}^{\infty} d m_{j+1} A\left(m_{\ell}, m_{j+1}\right) f_{j+1}\left(m_{1}, \ldots, m_{j+1}, t\right)+ \\
& +\frac{1}{2 V} \int_{0}^{\infty} d m_{j+1} \int_{0}^{\infty} d m_{j+2} A\left(m_{j+1}, m_{j+2}\right) f_{j+2}\left(m_{1}, \ldots, m_{j+2}, t\right) .
\end{aligned}
$$

By (3.14), (3.16), (3.17) and (3.18), it follows that $\left\{f_{j}(t)\right\}_{j \in \mathbb{N}^{*}}$ satisfy (3.13).

Only uniqueness remains to be proved. To this end we notice that, since $P_{N}(t) \equiv 0$ for every $N>N_{0}$, the same is true for the sequence $\left\{f_{j}(t)\right\}_{j \in \mathbb{N}^{*}}$, namely, $f_{j}(t) \equiv 0$ for $j>N_{0}$. Therefore the system (3.13) only contains a finite number of non trivial equations and, since the equation for $j=N_{0}$ only involves $f_{N_{0}}(t)$, the system decouples. We conclude using the same argument used in Theorem 2.4 to prove uniqueness for the mass distribution functions $\left\{P_{N}(t)\right\}_{N \in \mathbb{N}^{*}}$.

Remark 3.3 Suppose that the initial data $f_{0}$ also satisfies

$$
m_{0}=\int_{0}^{\infty} m f_{0}(m) d m<+\infty
$$

Since $f_{1}(t)$ is the density function associated to the average number of particles at time $t$ (cf. (3.2)), the quantity:

$$
\overline{M(t)}=\int_{0}^{\infty} m f_{1}(m, t) d m
$$

represents the average mass in the system at time $t$. If we integrate the equation for $j=1$ in system (3.13), a simple application of Fubini's theorem yields

$$
\frac{d}{d t} \int_{0}^{\infty} m f_{1}(m, t) d m=0 .
$$

Then, using (2.14) and (3.5)

$$
\overline{M(t)}=\overline{M(0)}=m_{0} N_{0} \quad \forall t>0 .
$$

This mass conservation property is a well known feature of the coagulation equation with bounded kernel. 


\section{The solution of the finite system for $\left\{f_{j}(t)\right\}_{j=1}^{N_{0}}$}

In this Section we study the behavior of the sequence $\left\{f_{j}(t)\right\}_{j=1}^{N_{0}}$ as $t \rightarrow+\infty$.

Proposition 4.1 Let $\left\{f_{j}(t)\right\}_{j=1}^{N_{0}}$ be the solution of (3.13) with initial datum (3.5) given by Corollary 3.1. Then:

(i) $\quad \exists N_{\infty} \in\left[1, N_{0}\right] ; \quad \lim _{t \rightarrow+\infty} \overline{N(t)}=N_{\infty}$.

(ii) $\quad \exists \sigma \geq 0: \lim _{t \rightarrow+\infty} \operatorname{var}(N)(t)=\sigma$.

(iii) If $A\left(m_{1} \cdot m_{2}\right) \geq C$ for some positive constant $C$ :

$$
\begin{aligned}
& \lim _{t \rightarrow+\infty} \overline{N(t)}=1, \\
& \lim _{t \rightarrow+\infty} \operatorname{var}(N)(t)=0,
\end{aligned}
$$

where $\operatorname{var}(N)(t)={\overline{(N(t))^{2}}}_{-\overline{N(t)}^{2}}$ is the variance of the distribution on particle numbers $P(t, N)$.

The proof of Proposition 4.1 follows from the three following auxiliary results.

Lemma 4.2 For all $t>0$ and $j \in \mathbb{N}$ :

$$
\begin{aligned}
\frac{d}{d t}\left\|f_{j}(t)\right\|_{L^{1}\left(\left(\mathbb{R}^{+}\right)^{j}\right)} & =-\frac{j}{2 V} \int d \mathbf{m}_{j+1} A\left(m_{1}, m_{2}\right) f_{j+1}\left(m_{j+1}, t\right)+ \\
& \quad-\frac{j(j-1)}{2 V} \int d \mathbf{m}_{j} A\left(m_{1}, m_{2}\right) f_{j}\left(m_{j}, t\right) \\
\leq & 0 .
\end{aligned}
$$

Proof of Lemma 4.2 Since, by definition, $f_{j}(t) \geq 0$ for any $j$ and $t$, Lemma 4.2 follows by a simple integration of $(3.13)$ with respect to $m_{1} \ldots m_{j}$.

From (3.2) and Lemma 4.2 we deduce

Corollary 4.3 (i) For all $t>0$ :

$$
\frac{d}{d t} \overline{N(t)} \leq 0
$$

(ii) There exists $N_{\infty} \in\left[1, N_{0}\right]$ such that $\overline{N(t)} \rightarrow N_{\infty}$ as $t \rightarrow+\infty$.

Proof of Corollary 4.3 Only the property $N_{\infty} \geq 1$ needs perhaps to be explained. From $(i)$ we deduce the existence of $N_{\infty} \in\left[0, N_{0}\right]$, the unique limit point of $\overline{N(t)}$ as $t \rightarrow+\infty$. On the other hand,

$$
\overline{N(t)}=\sum_{N=1}^{N_{0}} N P(t, N) \geq \sum_{N=1}^{N_{0}} P(t, N)=1,
$$

from where $N_{\infty} \geq 1$.

We also deduce: 
Corollary 4.4 (i) For all $t>0$ and every $j \geq 1$ :

$$
\overline{(N(t))(N(t)-1) \ldots(N(t)-j+1)} \leq N_{0}^{j} .
$$

(ii) For any $0<t_{1}<t_{2}$ and every $j \geq 1$ :

$$
\overline{\left(N\left(t_{2}\right)\right)\left(N\left(t_{2}\right)-1\right) \ldots\left(N\left(t_{2}\right)-j+1\right)} \leq \overline{\left(N\left(t_{1}\right)\right)\left(N\left(t_{1}\right)-1\right) \ldots\left(N\left(t_{1}\right)-j+1\right)} .
$$

(iii) If $A\left(m_{1} \cdot m_{2}\right) \geq C$ for some positive constant $C$, then for all $t>0$ and every $j \geq 1$ :

$$
\overline{(N(t))(N(t)-1) \ldots(N(t)-j+1)} \leq e^{-C \frac{j(j-1)}{2 V} t} N_{0}^{j} .
$$

Proof of Corollary 4.4 By Lemma 4.2 and (3.5)

$$
\left\|f_{j}(t)\right\|_{L^{1}\left(\left(\mathbb{R}^{+}\right)^{j}\right)} \leq\left\|f_{j}(0)\right\|_{L^{1}\left(\left(\mathbb{R}^{+}\right)^{j}\right)} \leq N_{0}^{j},
$$

for any time $t$. Since, by definition, $\overline{(N(t))(N(t)-1) \ldots(N(t)-j+1)}=\left\|f_{j}(t)\right\|_{L^{1}\left(\left(\mathbb{R}^{+}\right)^{j}\right)}$,

this concludes the proof of $(i)$ and $(i i)$. If, in order to prove (iii), we assume $A\left(m_{1}, m_{2}\right) \geq C>0$, then by Lemma 4.2 :

$$
\frac{d}{d t}\left\|f_{j}(t)\right\|_{L^{1}\left(\left(\mathbb{R}^{+}\right)^{j}\right)} \leq-C \frac{j(j-1)}{2 V}\left\|f_{j}(t)\right\|_{L^{1}\left(\left(\mathbb{R}^{+}\right)^{j}\right)} .
$$

By (3.5) we deduce

$$
\left\|f_{j}(t)\right\|_{L^{1}\left(\left(\mathbb{R}^{+}\right)^{j}\right)} \leq e^{-C \frac{j(j-1)}{2 V} t}\left\|f_{j}(0)\right\|_{L^{1}\left(\left(\mathbb{R}^{+}\right)^{j}\right)} \leq e^{-C \frac{j(j-1)}{2 V} t} N_{0}^{j},
$$

for all time $t$, and (iii) follows.

Proof of Proposition 4.1 Property ( $i$ ) follows from Corollary 4.3. To prove (ii) we notice first that, by Corollary 4.4 for $j=2$ :

$$
\left\|f_{2}(t)\right\|_{L^{1}\left(\left(\mathbb{R}^{+}\right)^{2}\right)}=\overline{(N(t))(N(t)-1)} \leq N_{0}^{2},
$$

and, by Lemma $4.2,\left\|f_{2}(t)\right\|_{L^{1}\left(\left(\mathbb{R}^{+}\right)^{2}\right)}$ is decreasing in time. Then there exists $G_{2} \in\left[0, N_{0}^{2}\right]$ such that:

$$
\left\|f_{2}(t)\right\|_{L^{1}\left(\left(\mathbb{R}^{+}\right)^{2}\right)} \rightarrow G_{2} \text {, as } t \rightarrow+\infty .
$$

Thus

$$
\lim _{t \rightarrow+\infty} \overline{(N(t))^{2}}=G_{2}+\lim _{t \rightarrow+\infty} \overline{N(t)}=G_{2}+N_{\infty}
$$

Therefore, the variance of the distribution on particle numbers for large times is given by:

$$
\lim _{t \rightarrow+\infty} \operatorname{var}(N)(t)=\lim _{t \rightarrow+\infty}\left(\overline{(N(t))^{2}}-\overline{N(t)}^{2}\right)=G_{2}+N_{\infty}-N_{\infty}^{2}:=\sigma .
$$


Proof of (iii). By Corollary 4.4 for $j=2$ :

$$
\left\|f_{2}(t)\right\|_{L^{1}\left(\left(\mathbb{R}^{+}\right)^{2}\right)}=\overline{(N(t))(N(t)-1)} \leq e^{-\frac{t C}{V}} N_{0}^{2} \rightarrow 0, \quad \text { as } t \rightarrow+\infty
$$

and then

$$
\lim _{t \rightarrow+\infty} \overline{(N(t))^{2}}=\lim _{t \rightarrow+\infty} \overline{N(t)}=N_{\infty}
$$

Therefore, the variance of the distribution on particle numbers for large times is given by:

$$
\lim _{t \rightarrow+\infty} \operatorname{var}(N)(t)=\lim _{t \rightarrow+\infty}\left(\overline{(N(t))^{2}}-\overline{N(t)}^{2}\right)=N_{\infty}-N_{\infty}^{2}
$$

Since, by definition, the variance is non-negative:

$$
N_{\infty}-N_{\infty}^{2} \geq 0 \Rightarrow N_{\infty}\left(1-N_{\infty}\right) \geq 0 \Rightarrow 0 \leq N_{\infty} \leq 1 .
$$

On the other hand by Corollary 4.3 we know that $N_{\infty} \geq 1$. It follows that $N_{\infty}=1$ and therefore

$$
\lim _{t \rightarrow+\infty} \operatorname{var}(N)(t)=0
$$

Remark 4.5 In the case (iii) of Proposition 4.1, the distribution of particle numbers, that is initially a Dirac measure, converges, as $t \rightarrow+\infty$, to another delta distribution, centered now at $N_{\infty}=1$. As expected, due to the coalescence dynamics, the system is finally formed by one single large particle, of mass $N_{0} m_{0}$.

\section{BBGKY hierarchy of the rescaled correlation functions}

We consider in this Section the limit of the finite particle system, when the volume $V$, and the initial number of particles $N_{0}$ go to infinity in such a way that:

$$
\lim _{V, N_{0} \rightarrow+\infty} \frac{N_{0}}{V}=\rho_{0} \in(0,+\infty) .
$$

To this end let us define the rescaled correlation functions $\left\{f_{j}^{V}(t)\right\}_{j=1}^{N_{0}}$ as

$$
f_{j}^{V}\left(m_{1}, \ldots, m_{j}, t\right):=\frac{f_{j}\left(m_{1}, \ldots, m_{j}, t\right)}{V^{j}}, \quad j=1, \ldots, N_{0} .
$$

Since, as we have seen in Section 3, the function $f_{1}$ is the number density function, the rescaled function $f_{1}^{V}$ is the density function associated to the concentration of particles (number of particles per unit volume). 
By (3.13) the functions $f_{j}^{V}$ satisfy the following set of $N_{0}$ equations:

$$
\begin{aligned}
\partial_{t} f_{j}^{V} & =\frac{1}{2} \sum_{\ell=1}^{j} \int_{0}^{m_{\ell}} d \mu A\left(m_{\ell}-\mu, \mu\right) f_{j+1}^{V}\left(m_{1}, \ldots, m_{\ell}-\mu, \ldots, m_{j}, \mu, t\right)+ \\
& -\frac{S\left(\mathbf{m}_{j}\right)}{2 V} f_{j}^{V}\left(m_{1}, \ldots, m_{j}, t\right)-\sum_{\ell=1}^{j} \int_{0}^{\infty} d \mu A\left(m_{\ell}, \mu\right) f_{j+1}^{V}\left(m_{1}, \ldots, m_{j}, \mu, t\right), \quad j \in \mathbb{N}^{*},
\end{aligned}
$$

for $j=1,2, \ldots, N_{0}$. We will refer to the family of equations (5.3) as BBGKY hierarchy by analogy with the system arising in the framework of classical particle systems.

By (3.5) and (5.2) the rescaled densities at time $t=0$ are

$$
f_{j}^{V}\left(m_{1}, \ldots, m_{j}, 0\right)=\frac{\left(N_{0}\right) !}{\left(N_{0}-j\right) !} \frac{1}{V^{j}} f_{0}^{\otimes j}\left(m_{1}, \ldots, m_{j}\right), \quad j=1,2, \ldots, N_{0},
$$

where the function $f_{0}$ has been defined in (2.14). By (5.4), for every $j \geq 1$ we have:

$$
\lim _{\substack{V, N_{0} \rightarrow+\infty \\ \frac{N_{0}}{V} \rightarrow \rho_{0}}}\left\|f_{j}^{V}(0)-\rho_{0}^{j} f_{0}^{\otimes j}\right\|_{L^{1}\left(\left(\mathbb{R}^{+}\right)^{j}\right)}=0 .
$$

In order to state our main result we first recall that, for all non negative initial data in $L^{1}\left(\mathbb{R}^{+}\right)$, the Cauchy problem for the coagulation equation (1.1) with bounded kernel has a unique non negative solution in $\mathbf{C}\left([0,+\infty) ; L^{1}\left(\mathbb{R}^{+}\right)\right.$) (cf.[47], Theorem 2.1 ).

Our main result is then the following:

Theorem 5.1 Let $\left\{f_{j}(t)\right\}_{j=1}^{N_{0}}$ be the solution of system (3.13) with initial data defined in (3.5). Then, if $\left\{f_{j}^{V}(t)\right\}_{j=1}^{N_{0}}$ is the sequence of rescaled densities defined by (5.2):

$$
\forall j \geq 1: \quad \lim _{\substack{V, N_{0} \rightarrow+\infty \\ \frac{N_{0}}{V} \rightarrow \rho_{0}}}\left\|f_{j}^{V}(t)-f(t)^{\otimes j}\right\|_{L^{1}\left(\left(\mathbb{R}^{+}\right)^{j}\right)}=0,
$$

where $f$ is the unique solution in $\mathbf{C}\left([0,+\infty) ; L^{1}\left(\mathbb{R}^{+}\right)\right)$of the coagulation equation (1.1) with kernel $A$ satisfying (2.12) and initial datum $\rho_{0} f_{0}, f_{0}$ given by (2.14).

The proof of Theorem 5.1 is done in two steps. The first is to show that the sequence $\left\{f_{j}^{V}(t)\right\}_{j=1}^{N_{0}}$ converges to a sequence of functions $\left\{f_{j}^{\infty}(t)\right\}_{j \in \mathbb{N}^{*}}$ that satisfy an infinite set of equations. It uses the explicit expression of the functions $f_{j}^{V}$ as a finite sum and a kind of dominated convergence. The second step is to prove that for all $j \geq 1$ and $t \geq 0, f_{j}^{\infty}(t)=f(t)^{\otimes j}$. This follows from the uniqueness of solutions of the new infinite system of equations. We start proving this uniqueness result.

For the sake of notation let us introduce operators $W_{j}$ defined as follows. Given a function $\varphi \in L^{1}\left(\left(\mathbb{R}^{+}\right)^{j+1}\right)$ we define $W_{j}[\varphi]$ as:

$$
W_{j}[\varphi]\left(\mathbf{m}_{j}\right):=V G_{j}[\varphi]\left(\mathbf{m}_{j}\right)-\sum_{\ell=1}^{j} \int_{0}^{\infty} d \mu A\left(m_{\ell}, \mu\right) \varphi\left(\mathbf{m}_{j}, \mu\right),
$$


where $G_{j}$ is defined by in (2.17). Using Lemma (2.3) it is easily seen that $W_{j}$ is a linear and continuous operator from $L^{1}\left(\left(\mathbb{R}^{+}\right)^{j+1}\right)$ to $L^{1}\left(\left(\mathbb{R}^{+}\right)^{j}\right)$ whose norm satisfies:

$$
\left\|W_{j}\right\| \leq \frac{3 j}{2} .
$$

Lemma 5.2 Let $f \in \mathbf{C}\left([0,+\infty) ; L^{1}\left(\mathbb{R}^{+}\right)\right)$be the unique non negative solution of the coagulation equation:

$$
\partial_{t} f(m, t)=\frac{1}{2} \int_{0}^{m} d \mu A(m-\mu, \mu) f(m-\mu, t) f(\mu, t)-f(m, t) \int_{0}^{\infty} d \mu A(m, \mu) f(\mu, t)
$$

with initial datum $f(0, m)=\rho_{0} f_{0}(m), f_{0}$ given by (2.14), and kernel A satisfying (2.12). Then, the sequence of functions defined as

$$
f_{j}^{\infty}\left(t, \mathbf{m}_{j}\right):=f(t)^{\otimes j}\left(\mathbf{m}_{j}\right), \quad j \in \mathbb{N}^{*}
$$

is the unique non negative solution of the system:

$$
\begin{aligned}
\frac{\partial f_{j}^{\infty}}{\partial t}\left(\mathbf{m}_{j}, t\right) & =\frac{1}{2} \sum_{\ell=1}^{j} \int_{0}^{m_{\ell}} d \mu A\left(m_{\ell}-\mu, \mu\right) f_{j+1}^{\infty}\left(m_{1}, \ldots, m_{\ell}-\mu, \ldots, m_{j}, \mu, t\right)+ \\
& -\sum_{\ell=1}^{j} \int_{0}^{\infty} d \mu A\left(m_{\ell}, \mu\right) f_{j+1}^{\infty}\left(m_{1}, \ldots, m_{j}, \mu, t\right), \text { for } j=1,2, \ldots
\end{aligned}
$$

with initial data $f_{j}^{\infty}(0)=\rho_{0}^{j} f_{0}^{\otimes j}$, such that $f_{j}^{\infty} \in \mathbf{C}\left([0,+\infty) ; L^{1}\left(\mathbb{R}^{+}\right)^{j}\right)$. For all $t>0$, the sequence $\left\{f_{j}^{\infty}(t)\right\}_{j \in \mathbb{N}^{*}}$ satisfies:

$$
\forall j \geq 1: \quad\left\|f_{j}^{\infty}(t)\right\|_{L^{1}\left(\left(\mathbb{R}^{+}\right)^{j}\right)} \leq \rho_{0}^{j} .
$$

Proof of Lemma 5.2 A straightforward calculation shows that the sequence $\left\{f_{j}^{\infty}(t)\right\}_{j \in \mathbb{N}^{*}}$ defined in (5.10) is indeed a solution of system (5.11) with initial data $\left\{\rho_{0}^{j} f_{0}^{\otimes j}\left(\mathbf{m}_{j}\right)\right\}_{j \in \mathbb{N}^{*}}$.

On the other hand, since $f \in \mathbf{C}\left([0,+\infty) ; L^{1}\left(\mathbb{R}^{+}\right)\right)$satisfies (5.9) we have $f \in \mathbf{C}^{1}\left((0,+\infty) ; L^{1}\left(\mathbb{R}^{+}\right)\right)$ and then $f_{j}^{\infty} \in \mathbf{C}\left([0,+\infty) ; L^{1}\left(\mathbb{R}^{+}\right)^{j}\right) \cap \mathbf{C}^{1}\left((0,+\infty) ; L^{1}\left(\mathbb{R}^{+}\right)^{j}\right)$. Since $\left\{f_{j}^{\infty}(t)\right\}_{j \in \mathbb{N}^{*}}$ satisfies (5.11), we obtain, after integration of the $j$-th equation over $\left(\mathbb{R}^{+}\right)^{j}$ :

$$
\frac{\partial}{\partial t}\left\|f_{j}^{\infty}(t)\right\|_{L^{1}\left(\left(\mathbb{R}^{+}\right)^{j}\right)}=-\frac{j}{2} \int d \mathbf{m}_{j+1} A\left(m_{1}, m_{2}\right) f_{j+1}^{\infty}(t) \leq 0 .
$$

Then, for all $t>0$ :

$$
\left\|f_{j}^{\infty}(t)\right\|_{L^{1}\left(\left(\mathbb{R}^{+}\right)^{j}\right)} \leq\left\|f_{j}^{\infty}(0)\right\|_{L^{1}\left(\left(\mathbb{R}^{+}\right)^{j}\right)}=\rho_{0}^{j},
$$

and that proves (5.12).

In order to prove uniqueness notice that the system (5.11) may be written using operator $W_{j}$ as

$$
\frac{\partial f_{j}^{\infty}}{\partial t}=W_{j}\left[f_{j}^{\infty}\right]
$$


from where

$$
f_{j}^{\infty}(t)=f_{j}^{\infty}(0)+\int_{0}^{t} d t_{1} W_{j} f_{j+1}^{\infty}\left(t_{1}\right) .
$$

Using this same formula for $f_{j+1}^{\infty}$, we obtain after $M$ iterations:

$$
\begin{aligned}
f_{j}^{\infty}(t) & =\sum_{n \geq 0}^{M} \frac{t^{n}}{n !} W_{j} W_{j+1} \ldots W_{j+n-1} f_{j+n}^{\infty}(0)+ \\
& +\int_{0}^{t} d t_{1} \ldots \int_{0}^{t_{M}} d t_{M+1} W_{j} \ldots W_{j+M} f_{j+M+1}^{\infty}\left(\mathbf{m}_{j+M+1} ; t_{M+1}\right) .
\end{aligned}
$$

Let us assume now that there exist two different non-negative solutions, $\left\{h_{j, k}(t)\right\}_{j \in \mathbb{N}^{*}}, k=1,2$, of (5.11) such that $h_{j, k} \in \mathbf{C}\left([0,+\infty) ; L^{1}\left(\mathbb{R}^{+}\right)^{j}\right)$ with the same factorized initial datum $\left\{\rho_{0}^{j}\left(f_{0}\right)^{\otimes j}\right\}_{j \in \mathbb{N}^{*}}$. Then by (5.15) and (5.8) we deduce that for every $M>0$ :

$$
\begin{aligned}
& \left\|h_{j, 1}(t)-h_{j, 2}(t)\right\|_{L^{1}\left(\left(\mathbb{R}^{+}\right)^{j}\right)} \leq \frac{t^{M+1}}{(M+1) !} \times \\
& \quad \times\left\|W_{j} \ldots W_{j+M}\left(h_{j+M+1,1}\left(t_{M+1}\right)-h_{j+M+1,2}\left(t_{M+1}\right)\right)\right\|_{L^{1}\left(\left(\mathbb{R}^{+}\right)^{j+M+1}\right)} \\
& \leq \frac{t^{M+1}}{(M+1) !}\left(\frac{3}{2}\right)^{M+1} \frac{(j+M) !}{(j-1) !}\left(\left\|h_{j+M+1,1}\left(t_{M+1}\right)\right\|_{L^{1}\left(\left(\mathbb{R}^{+}\right)^{j+M+1}\right)}+\right. \\
& \left.\quad+\left\|h_{j+M+1,2}\left(t_{M+1}\right)\right\|_{L^{1}\left(\left(\mathbb{R}^{+}\right)^{j+M+1}\right)}\right) .
\end{aligned}
$$

By our assumptions on $\left\{h_{j, k}(t)\right\}_{j \in \mathbb{N}^{*}}, k=1,2$, they both satisfy (5.12). Thus, plugging (5.12) into (5.16) we deduce:

$$
\left\|h_{j, 1}(t)-h_{j, 2}(t)\right\|_{L^{1}\left(\left(\mathbb{R}^{+}\right)^{j}\right)} \leq 2 \rho_{0}^{j+M+1} \frac{t^{M+1}}{(M+1) !}\left(\frac{3}{2}\right)^{M+1} \frac{(j+M) !}{(j-1) !} .
$$

Using (3.11) we obtain

$$
\left\|h_{j, 1}(t)-h_{j, 2}(t)\right\|_{L^{1}\left(\left(\mathbb{R}^{+}\right)^{j}\right)} \leq 2^{j} \rho_{0}^{j}\left(3 \rho_{0} t\right)^{M+1}
$$

and then, for every $t<1 /\left(3 \rho_{0}\right)$ :

$$
\left\|h_{j, 1}(t)-h_{j, 2}(t)\right\|_{L^{1}\left(\left(\mathbb{R}^{+}\right)^{j}\right)} \leq \lim _{M \rightarrow+\infty} 2^{j} \rho_{0}^{j}\left(3 \rho_{0} t\right)^{M+1}=0 .
$$

This shows the uniqueness but only for $t \in\left[0,1 / 3 \rho_{0}\right)$. This argument may be now iterated as follows. Suppose that (5.19) holds for $t \in[0, T)$ for some $T>0$. Consider now (5.16) for $t \in$ $\left[T, T+1 / 3 \rho_{0}\right.$ ) (since it holds for all $t>0$ ). Since (5.12) holds also true for all $t>0$, it follows as before that (5.19) also holds for $t \in\left[T, T+1 / 3 \rho_{0}\right)$. Therefore, global uniqueness is proven. This ends the proof of Lemma 5.2. 
Lemma 5.3 Under the same hypothesis than in Theorem 5.1 for all $T>0$ and all $j \geq 1$ :

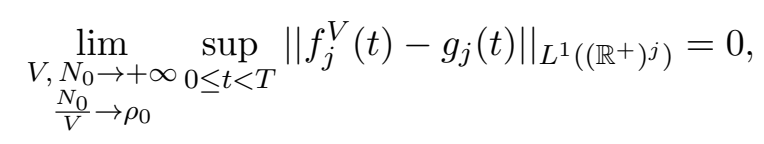

where

$$
g_{j}\left(t, \mathbf{m}_{j}\right)=\sum_{n=0}^{\infty} \rho_{0}^{j+n} \frac{t^{n}}{n !} W_{j} \circ W_{j+1} \cdots \circ W_{j+n-1}\left[f_{0}^{\otimes j+n}\right]\left(\mathbf{m}_{j}\right)
$$

is such that $g_{j} \in \mathbf{C}\left([0,+\infty) ; L^{1}\left(\mathbb{R}^{+}\right)^{j}\right) \cap \mathbf{C}^{1}\left((0, \infty) ; L^{1}\left(\mathbb{R}^{+}\right)^{j}\right)$ and $g_{j} \geq 0$. Moreover the sequence $\left\{g_{j}\right\}_{j \in \mathbb{N}^{*}}$ satisfies system (5.11) for $t \in(0, \infty)$.

Proof of Lemma 5.3 Let us prove first that the series in (5.21) defines a function $g_{j} \in$ $\mathbf{C}\left(\left[0, \tau_{1}\right) ; L^{1}\left(\mathbb{R}^{+}\right)^{j}\right) \cap \mathbf{C}^{1}\left(\left(0, \tau_{1}\right) ; L^{1}\left(\mathbb{R}^{+}\right)^{j}\right)$ for some $\tau_{1}>0$. To this end we first deduce the following estimate from (5.8) and the the explicit expression (5.4) of the initial datum:

$$
\begin{aligned}
& \left\|W_{j} \circ \ldots \circ W_{j+n-1}\left[f_{0}^{\otimes j+n}\right]\right\|_{L^{1}\left(\left(\mathbb{R}^{+}\right)^{j}\right)} \leq\left(\frac{3}{2}\right)^{n} j(j+1) \ldots(j+n-1)\left\|f_{0}\right\|_{L^{1}\left(\mathbb{R}^{+}\right)}^{j+n} \\
& =\left(\frac{3}{2}\right)^{n} j(j+1) \ldots(j+n-1) .
\end{aligned}
$$

Using again (3.11) we deduce, for all $j \geq 1, n \geq 0$ and $t>0$ :

$$
\rho_{0}^{j+n} \frac{t^{n}}{n !}\left\|W_{j} \circ \cdots \circ W_{j+n-1}\left[f_{0}^{\otimes j+n}\right]\right\|_{L^{1}\left(\left(\mathbb{R}^{+}\right)^{j}\right)} \leq 2^{j-1} \rho_{0}^{j}\left(3 \rho_{0} t\right)^{n} .
$$

It then follows that the series in (5.21) defines a continuous function $g_{j} \in \mathbf{C}\left(\left[0, \frac{1}{3 \rho_{0}}\right) ; L^{1}\left(\left(\mathbb{R}^{+}\right)^{j}\right)\right)$. A similar argument shows that $g_{j} \in \mathbf{C}^{1}\left(\left(0, \frac{1}{3 \rho_{0}}\right) ; L^{1}\left(\left(\mathbb{R}^{+}\right)^{j}\right)\right)$ and

$$
\frac{\partial g_{j}(t)}{\partial t}=\sum_{n=1}^{\infty} \rho_{0}^{j+n} \frac{t^{n-1}}{(n-1) !} W_{j} \circ W_{j+1} \cdots \circ W_{j+n-1}\left[f_{0}^{\otimes j+n}\right]\left(\mathbf{m}_{j}\right) .
$$

By construction the sequence $\left\{f_{j}^{V}(t)\right\}_{j=1}^{N_{0}}$ satisfies, for every $t>0$ :

$$
\begin{aligned}
& f_{j}^{V}\left(t, \mathbf{m}_{j}\right)=e^{-\frac{S\left(\mathbf{m}_{j}\right)}{2 V} t} f_{j}^{V}\left(\mathbf{m}_{j} ; 0\right)-\int_{0}^{t} d t_{1} \sum_{\ell=1}^{j} \int_{0}^{\infty} d \mu e^{-\frac{S\left(\mathbf{m}_{j}\right)}{2 V}\left(t-t_{1}\right)} A\left(m_{\ell}, \mu\right) f_{j+1}^{V}\left(\mathbf{m}_{j}, \mu ; t_{1}\right)+ \\
& +\frac{1}{2} \int_{0}^{t} d t_{1} \sum_{\ell=1}^{j} \int_{0}^{m_{\ell}} d \mu e^{-\frac{S\left(\mathbf{m}_{j}\right)}{2 V}\left(t-t_{1}\right)} A\left(m_{\ell}-\mu, \mu\right) f_{j+1}^{V}\left(m_{1}, \ldots, m_{\ell}-\mu, \ldots, m_{j}, \mu ; t_{1}\right) .
\end{aligned}
$$

Using the operator $W_{j}$ defined by (5.7), this equation can be rewritten as follows:

$$
\begin{aligned}
f_{j}^{V}(t)= & e^{-\frac{S\left(\mathbf{m}_{j}\right)}{2 V} t} f_{j}^{V}(0)+\int_{0}^{t} d t_{1} e^{-\frac{S\left(\mathbf{m}_{j}\right)}{2 V}\left(t-t_{1}\right)} W_{j} f_{j+1}^{V}\left(t_{1}\right)= \\
= & \sum_{n \geq 0}^{N_{0}-j} \int_{0}^{t} d t_{1} \ldots \int_{0}^{t_{n-1}} d t_{n} e^{-\frac{S\left(\mathbf{m}_{j}\right)}{2 V}\left(t-t_{1}\right)} W_{j}\left[e^{-\frac{S\left(\mathbf{m}_{j+1}\right)}{2 V}\left(t_{1}-t_{2}\right)} W_{j+1}[\ldots\right. \\
& \left.\ldots e^{-\frac{S\left(\mathbf{m}_{j+n-1}\right)}{2 V}\left(t_{n-1}-t_{n}\right)} W_{j+n-1}\left[e^{-\frac{S\left(\mathbf{m}_{j+n}\right)}{2 V} t_{n}} f_{j+n}^{V}(0)\right] \ldots\right],
\end{aligned}
$$


where we set $t_{-1} \equiv 0, t_{0} \equiv t$.

Property (5.20) follows from the two following facts. The first is that the sum:

$$
\begin{aligned}
& \sum_{n \geq 0}^{N_{0}-j} \int_{0}^{t} d t_{1} \ldots \int_{0}^{t_{n-1}} d t_{n} \| e^{-\frac{S\left(\mathbf{m}_{j}\right)}{2 V}\left(t-t_{1}\right)} W_{j}\left[e^{-\frac{S\left(\mathbf{m}_{j+1}\right)}{2 V}\left(t_{1}-t_{2}\right)} W_{j+1}[\ldots\right. \\
& \left.\ldots e^{-\frac{S\left(\mathbf{m}_{j+n-1}\right)}{2 V}\left(t_{n-1}-t_{n}\right)} W_{j+n-1}\left[e^{-\frac{S\left(\mathbf{m}_{j+n}\right)}{2 V} t_{n}} f_{j+n}^{V}(0)\right] \ldots\right] \|_{L^{1}\left(\left(\mathbb{R}^{+}\right)^{j}\right)}
\end{aligned}
$$

is dominated by a convergent series uniformly for $t \in\left[0,1 / 6 \rho_{0}\right)$. The second is that for every $j \geq 1$ and every $n \geq 1$ :

$$
\begin{gathered}
\lim _{\substack{V, N_{0} \rightarrow+\infty \\
\frac{N_{0}}{V} \rightarrow \rho_{0}}}\left(\int d \mathbf { t } _ { n } e ^ { - \frac { S ( \mathbf { m } _ { j } ) } { 2 V } ( t - t _ { 1 } ) } W _ { j } \left[e^{-\frac{S\left(\mathbf{m}_{j+1}\right)}{2 V}\left(t_{1}-t_{2}\right)} W_{j+1}[\ldots\right.\right. \\
\left.\left.\cdots e^{-\frac{S\left(\mathbf{m}_{j+n-1}\right)}{2 V}\left(t_{n-1}-t_{n}\right)} W_{j+n-1}\left[e^{-\frac{S\left(\mathbf{m}_{j+n}\right)}{2 V} t_{n}} f_{j+n}^{V}(0)\right] \ldots\right]\right) \\
=\rho_{0}^{j+n} \frac{t^{n}}{n !} W_{j} \circ W_{j+1} \cdots \circ W_{j+n-1}\left[f_{0}^{\otimes j+n}\right]
\end{gathered}
$$

in $L^{1}\left(\left(\mathbb{R}^{+}\right)^{j}\right)$, uniformly for $t \in[0, T]$ for any $T>0$.

We start proving the first. By (5.4) and (5.8) we obtain:

$$
\begin{aligned}
& \| W_{j}\left[e ^ { - \frac { S ( \mathbf { m } _ { j } ) } { 2 V } ( t - t _ { 1 } ) } W _ { j + 1 } \left[e^{-\frac{S\left(\mathbf{m}_{j+1}\right)}{2 V}\left(t_{1}-t_{2}\right)} \ldots\right.\right. \\
& \left.\left.\ldots W_{j+n-1}\left[e^{-\frac{S\left(\mathbf{m}_{j+n-1}\right)}{2 V}\left(t_{n-1}-t_{n}\right)} e^{-\frac{S\left(\mathbf{m}_{j+n}\right)}{2 V} t_{n}} f_{j+n}^{V}(0)\right]\right] \ldots\right] \|_{\left.L^{1}\left(\mathbb{R}^{+}\right)^{j}\right)} \\
& \leq\left(\frac{3}{2}\right)^{n} j(j+1) \ldots(j+n-1)\left\|f_{j+n}^{V}(0)\right\|_{L^{1}\left(\left(\mathbb{R}^{+}\right)^{j}\right)} \\
& \leq\left(\frac{3}{2}\right)^{n} j(j+1) \ldots(j+n-1)\left(\frac{N_{0}}{V}\right)^{j+n} .
\end{aligned}
$$

Since in the expression (5.25) $t_{n}<t_{n-1}<\cdots<t_{1}<t_{0} \equiv t$, it follows that $t_{k-1}-t_{k}>0$ for any $k=1, \ldots, n$ we deduce:

$$
\begin{aligned}
& \int d \mathbf{t}_{n} \| e^{-\frac{S\left(\mathbf{m}_{j}\right)}{2 V}\left(t-t_{1}\right)} W_{j}\left[e^{-\frac{S\left(\mathbf{m}_{j+1}\right)}{2 V}\left(t_{1}-t_{2}\right)} W_{j+1}[\ldots\right. \\
& \left.\ldots e^{-\frac{S\left(\mathbf{m}_{j+n-1}\right)}{2 V}\left(t_{n-1}-t_{n}\right)} W_{j+n-1}\left[e^{-\frac{S\left(\mathbf{m}_{j+n}\right)}{2 V} t_{n}} f_{j+n}^{V}(0)\right] \ldots\right] \mid \|_{L^{1}\left(\left(\mathbb{R}^{+}\right)^{j}\right)} \\
& \leq \frac{t^{n}}{n !}\left(\frac{3}{2}\right)^{n} j(j+1) \ldots(j+n-1)\left(\frac{N_{0}}{V}\right)^{j+n} .
\end{aligned}
$$


Using again (3.11) and the hypothesis (5.1) we deduce, for all $j \geq 1, n \geq 0$ and $t>0$ :

$$
\begin{array}{cc}
\int d \mathbf{t}_{n} \| e^{-\frac{S\left(\mathbf{m}_{j}\right)}{2 V}\left(t-t_{1}\right)} W_{j}\left[e^{-\frac{S\left(\mathbf{m}_{j+1}\right)}{2 V}\left(t_{1}-t_{2}\right)} W_{j+1}[\ldots\right. & \\
\ldots e^{-\frac{S\left(\mathbf{m}_{j+n-1}\right)}{2 V}\left(t_{n-1}-t_{n}\right)} W_{j+n-1}\left[e^{-\frac{S\left(\mathbf{m}_{j+n}\right)}{2 V} t_{n}}\right. & \left.\left.f_{j+n}^{V}(0)\right] \ldots\right] \\
& \leq 4^{j-1}\left(6 \rho_{0} t\right)^{n} .
\end{array}
$$

Estimate (5.29) shows that the sum giving $f_{j}^{V}(t)$ in (5.25) is dominated, uniformly for $t \in\left[0, \tau_{1}\right)$, $\tau_{1}=\frac{1}{6 \rho_{0}}$, by a convergent series.

Property (5.26) for every $j \geq 1$ and every $n \geq 1$ follows from (5.5), the continuity of the operators $W_{k}$ and the uniform convergence of $e^{-\frac{S\left(\mathbf{m}_{k}\right)}{2 V} \tau}$ to 1 for $\mathbf{m}_{k} \in\left(\mathbb{R}^{+}\right)^{k}$ and $\tau \in\left[0, \tau_{1}\right)$.

Therefore:

$$
f_{j}^{V}(t) \rightarrow \sum_{n \geq 0} \frac{t^{n}}{n !} \rho_{0}^{j+n} W_{j} \circ \cdots \circ W_{j+n-1}\left[f_{0}^{\otimes j+n}\right] \equiv g_{j}(t)
$$

in $\mathbf{C}\left(\left[0, \tau_{1}\right) ; L^{1}\left(\mathbb{R}^{+}\right)\right) \cap \mathbf{C}^{1}\left(\left(0, \tau_{1}\right) ; L^{1}\left(\mathbb{R}^{+}\right)\right)$. Since $f_{j}^{V}(t) \geq 0$ for all $j \in \mathbb{N}^{*}$ and $t \geq 0$ it follows that $g_{j}(t) \geq 0$ for $t \in\left[0, \tau_{1}\right)$ and all $j$.

A straightforward computation shows that $\left\{g_{j}\right\}_{j \in N^{*}}$ satisfies system (5.11) for $t \in\left[0, \tau_{1}\right)$ and this proves the Lemma 5.3 for $T \in\left[0, \tau_{1}\right)$.

In order to extend this result for $T>\tau_{1}$ we first notice that for all $t>0$ :

$$
\begin{aligned}
f_{j}^{V}\left(t+\frac{\tau_{1}}{2}\right)= & e^{-\frac{S\left(\mathbf{m}_{j}\right)}{2 V} t} f_{j}^{V}\left(\tau_{1} / 2\right)+\int_{0}^{t} d t_{1} e^{-\frac{S\left(\mathbf{m}_{j}\right)}{2 V}\left(t-t_{1}\right)} W_{j}\left[f_{j+1}^{V}\left(t_{1}+\frac{\tau_{1}}{2}\right)\right] \\
= & \sum_{n \geq 0}^{N_{0}-j} \int_{0}^{t} d t_{1} \ldots \int_{0}^{t_{n-1}} d t_{n} e^{-\frac{S\left(\mathbf{m}_{j}\right)}{2 V}\left(t-t_{1}\right)} W_{j}\left[e^{-\frac{S\left(\mathbf{m}_{j+1}\right)}{2 V}\left(t_{1}-t_{2}\right)} W_{j+1}[\ldots\right. \\
& \left.\ldots e^{-\frac{S\left(\mathbf{m}_{j+n-1}\right)}{2 V}\left(t_{n-1}-t_{n}\right)} W_{j+n-1}\left[e^{-\frac{S\left(\mathbf{m}_{j+n}\right)}{2 V} t_{n}} f_{j+n}^{V}\left(\tau_{1} / 2\right)\right] \ldots\right] .
\end{aligned}
$$

In order to pass to the limit as $V \rightarrow+\infty$ and $N_{0} / V \rightarrow \rho_{0}$ in (5.30) we use the same two arguments as for (5.25). More precisely, using Lemma 4.2, we obtain:

$$
\begin{aligned}
& \| e^{-\frac{S\left(\mathbf{m}_{j}\right)}{2 V}\left(t-t_{1}\right)} W_{j}\left[e^{-\frac{S\left(\mathbf{m}_{j+1}\right)}{2 V}\left(t_{1}-t_{2}\right)} W_{j+1}[\ldots\right. \\
& \left.\ldots e^{-\frac{S\left(\mathbf{m}_{j+n-1}\right)}{2 V}\left(t_{n-1}-t_{n}\right)} W_{j+n-1}\left[e^{-\frac{S\left(\mathbf{m}_{j+n}\right)}{2 V} t_{n}} f_{j+n}^{V}\left(\tau_{1} / 2\right)\right] \ldots\right] \\
& \left.\leq\left(\frac{3}{2}\right)^{n} j(j+1) \ldots(j+n-1)\left\|f_{j+n}^{V}(0)\right\|_{L^{1}\left(\left(\mathbb{R}^{+}\right)^{j}\right)}\left(\mathbb{R}^{+}\right)^{j}\right) \\
& \leq\left(\frac{3}{2}\right)^{n} j(j+1) \ldots(j+n-1)\left(\frac{N_{0}}{V}\right)^{j+n}
\end{aligned}
$$


and then, arguing as before:

$$
\begin{aligned}
& \int d \mathbf{t}_{n} \| e^{-\frac{S\left(\mathbf{m}_{j}\right)}{2 V}\left(t-t_{1}\right)} W_{j}\left[e^{-\frac{S\left(\mathbf{m}_{j+1}\right)}{2 V}\left(t_{1}-t_{2}\right)} W_{j+1}[\ldots\right. \\
& \left.\ldots e^{-\frac{S\left(\mathbf{m}_{j+n-1}\right)}{2 V}\left(t_{n-1}-t_{n}\right)} W_{j+n-1}\left[e^{-\frac{S\left(\mathbf{m}_{j+n}\right)}{2 V} t_{n}} f_{j+n}^{V}\left(\tau_{1} / 2\right)\right] \ldots\right] \|_{L^{1}\left(\left(\mathbb{R}^{+}\right)^{j}\right)} \\
& \leq 4^{j-1}\left(6 \rho_{0} t\right)^{n} \text {. }
\end{aligned}
$$

The uniform convergence of $e^{-\frac{S\left(\mathbf{m}_{k}\right)}{2 V} \tau}$ to 1 for $\mathbf{m}_{k} \in\left(\mathbb{R}^{+}\right)^{k}$ and $\tau \in\left[0, \tau_{1}\right)$, the continuity of the operators $W_{k}$ and the convergence of $f_{j+n}^{V}\left(\tau_{1} / 2\right)$ to $f_{j+n}^{V}\left(\tau_{1} / 2\right)$ in $L^{1}\left(\mathbb{R}^{j+n}\right)$ that has just been proved before show that, as $V \rightarrow \infty, N_{0} / V \rightarrow \rho_{0}$ :

$$
\begin{aligned}
& f_{j}^{V}\left(t+\frac{\tau_{1}}{2}\right) \rightarrow h_{j}(t) \quad \text { in } \mathbf{C}\left(\left[0, \tau_{1}\right) ; L^{1}\left(\mathbb{R}^{+}\right)\right) \cap \mathbf{C}^{1}\left(\left(0, \tau_{1}\right) ; L^{1}\left(\mathbb{R}^{+}\right)\right) \\
& h_{j}(t)=\sum_{n \geq 0} \frac{t^{n}}{n !} \rho_{0}^{j+n} W_{j} \circ \cdots \circ W_{j+n-1}\left[g_{j}\left(\tau_{1} / 2\right)\right] \geq 0 \quad \forall t \in\left[0, \tau_{1}\right) .
\end{aligned}
$$

We define now the function $g_{j}$ for $t \in\left[\tau_{1}, 3 \tau_{1} / 2\right)$ as :

$$
g_{j}(t)=h_{j}\left(t-\tau_{1} / 2\right), \forall t \in\left[\tau_{1}, 3 \tau_{1} / 2\right) .
$$

Since $\left\{g_{j}(t)\right\}_{j \in N^{*}}$ satisfies system (5.11) for $t \in\left[0, \tau_{1}\right)$ it is such that:

$$
g\left(t+\frac{\tau_{1}}{2}\right)=\sum_{n \geq 0} \frac{t^{n}}{n !} \rho_{0}^{j+n} W_{j} \circ \cdots \circ W_{j+n-1}\left[g_{j}\left(\tau_{1} / 2\right)\right] \quad \forall t \in\left[0, \tau_{1} / 2\right) .
$$

It then follows $h_{j}(t)=g_{j}\left(t+\tau_{1} / 2\right)$ for all $t \in\left[0, \tau_{1} / 2\right)$. We deduce that $g_{j} \in \mathbf{C}\left(\left[0,3 \tau_{1} / 2\right) ; L^{1}\left(\mathbb{R}^{+}\right)\right) \cap$ $\mathbf{C}^{1}\left(\left(0,3 \tau_{1} / 2\right) ; L^{1}\left(\mathbb{R}^{+}\right)\right)$and

$$
\begin{aligned}
& f_{j}^{V}(t) \rightarrow g_{j}(t) \quad \text { in } \mathbf{C}\left(\left[0,3 \tau_{1} / 2\right) ; L^{1}\left(\mathbb{R}^{+}\right)\right) \cap \mathbf{C}^{1}\left(\left(0,3 \tau_{1} / 2\right) ; L^{1}\left(\mathbb{R}^{+}\right)\right), \\
& \text {as } N_{0} \rightarrow \infty, V \rightarrow \infty, \frac{N_{0}}{V} \rightarrow \rho_{0} .
\end{aligned}
$$

Since, as a plain calculation shows again, the sequence $\left\{h_{j}(t)\right\}_{j \in \mathbb{N}^{*}}$ satisfies system (5.11) for $t \in\left[0, \tau_{1}\right)$ and $h_{j}(0)=g_{j}\left(\tau_{1} / 2\right)$ the sequence $\left\{g_{j}\right\}_{j \in \mathbb{N}^{*}}$ satisfies system $(5.11)$ for $t \in\left[0,3 \tau_{1} / 2\right)$.

We have extended in that way the previous result on $\left[0, \tau_{1}\right)$ to the interval $\left[0,3 \tau_{1} / 2\right)$. This procedure may be repeated to obtain the result in all finite interval $[0, T)$ from where Lemma 5.3 follows.

Proof of Theorem 5.1 From Lemma 5.3, for any $T>0, f_{j}^{V}$ converges to $g_{j}$ in $\mathbf{C}\left([0, T) ; L^{1}\left(\mathbb{R}^{+}\right)^{j}\right)$ and $\left\{g_{j}\right\}_{j \in N^{*}}$ is a non negative solution of system (5.11) with initial data $\left\{\rho_{0}^{j} f_{0}^{\otimes j}\right\}_{j \in \mathbb{N}^{*}}$. By Lemma 5.2 we have $g_{j}=f_{j}^{\infty}$ for all $j \geq 1$ and Theorem 5.1 follows. 
Remark 5.4 We have considered a factorized initial data for the finite system of particles (cf. (2.13)). But we could also have considered a more general initial data of the form:

$$
P_{N_{0}}\left(\mathbf{m}_{N_{0}}, 0\right)=\left(N_{0}\right) ! \Psi_{N_{0}}\left(\mathbf{m}_{N_{0}}\right), \quad P_{N}\left(\mathbf{m}_{N}, 0\right)=0 \text { for } \quad N \neq N_{0},
$$

with

$$
\Psi_{N_{0}}\left(\mathbf{m}_{N_{0}}\right) \geq 0, \quad \int d \mathbf{m}_{N_{0}} \Psi_{N_{0}}\left(\mathbf{m}_{N_{0}}\right)=1
$$

Then, for any $j=1, \ldots, N_{0}$, the correlation functions at time $t=0$ would have been

$$
f_{j}\left(\mathbf{m}_{j}, 0\right)=\sum_{N=j}^{\infty} \frac{1}{(N-j) !} \int d m_{j+1} \ldots \int d m_{N} P_{N}\left(\mathbf{m}_{N}, 0\right)=\frac{\left(N_{0}\right) !}{\left(N_{0}-j\right) !} \quad F_{j}^{\left(N_{0}\right)}\left(\mathbf{m}_{j}\right),
$$

where $F_{j}^{\left(N_{0}\right)}\left(\mathbf{m}_{j}\right)$ is the $j$-particle marginal distribution (in the sense of probability measures) of $P_{N_{0}}\left(\mathbf{m}_{N_{0}}\right)$, namely:

$$
F_{j}^{\left(N_{0}\right)}\left(\mathbf{m}_{j}\right):=\int d m_{j+1} \ldots \int d m_{N_{0}} \Psi_{N_{0}}\left(\mathbf{m}_{N_{0}}\right)
$$

and, by definition (see (5.33)):

$$
\int d \mathbf{m}_{j} F_{j}^{\left(N_{0}\right)}\left(\mathbf{m}_{j}\right)=\int d \mathbf{m}_{j} \int d m_{j+1} \ldots \int d m_{N_{0}} \Psi_{N_{0}}\left(\mathbf{m}_{N_{0}}\right)=1 .
$$

Then, the rescaled correlation functions at time $t=0$ would have satisfied

$$
\lim _{\substack{V, N_{0} \rightarrow+\infty \\ \frac{N_{0}}{V} \rightarrow \rho_{0}}}\left\|f_{j}^{V}(0)\right\|_{L^{1}\left(\left(\mathbb{R}^{+}\right)^{j}\right)}=\rho_{0}^{j} .
$$

Now, assuming that the functions $F_{j}^{\left(N_{0}\right)}(0)$ satisfy

$$
\lim _{N_{0} \rightarrow+\infty}\left\|F_{j}^{\left(N_{0}\right)}(0)-f_{0}^{\otimes j}\right\|_{L^{1}\left(\left(\mathbb{R}^{+}\right)^{j}\right)}=0
$$

for some $f_{0}(m)$ such that

$$
f_{0}(m) \geq 0, \quad \int d m f_{0}(m)=1,
$$

we could have proved exactly the same results that we got for the a priori factorized case.

Choosing an intial datum as in (5.36) would have meant to assume propagation of chaos to hold at time $t=0$. On the other hand, what we did has been to assume hypotheses of molecular chaos to hold at time $t=0$.

Acknowledgements. M. E. is supported by Grants MTM2008-03541 and IT-305-07. F. P. is supported by Grant MTM 2007-62186 and IT-305-07. F.P. acknowledges the UPV/EHU where most of this work was done as she held a post doctoral position. The authors thank the referee for his valuable comments and indications. 


\section{References}

[1] A. S. Ackleh, B. G. Fitzpatrick, and T. G. Hallam, Approximation and parameter estimation problems for algal aggregation models, Math. Models Methods Appl. Sci., 4 (1994), pp. 291-311.

[2] D. J. Aldous, Deterministic and stochastic models for coalescence (aggregation and coagulation): a review of the mean-field theory for probabilists, Bernoulli, 5 (1999), pp. 3-48.

[3] E. J. Allen And P. Bastien, On coagulation and the stellar mass function, Astrophys. J., 452 (1995), pp. 652-670.

[4] N. Bellomo, A. Bellouquid, J. Nieto, and J. Soler, On the asymptotic theory from microscopic to macroscopic growing tissue models: an overview with perspectives, Math. Models Methods Appl. Sci., 22, 1130001 (2012).

[5] N. Bellomo, C. Bianca, And M. Delitala, Complexity analysis and mathematical tools towards the modelling of living systems, Physics of Life Reviews, 6 (2009), pp. $144-175$.

[6] N. Bellomo and M. Delitala, From the mathematical kinetic, and stochastic game theory to modelling mutations, onset, progression and immune competition of cancer cells, Physics of Life Reviews, 5 (2008), pp. 183 - 206.

[7] N. Bellomo And G. Forni, Looking for new paradigms towards a biological-mathematical theory of complex multicellular systems, Math. Models Methods Appl. Sci., 16 (2006), pp. 1001-1029.

[8] A. Bellouquid and C. Bianca, Modelling aggregation-fragmentation phenomena from kinetic to macroscopic scales, Mathematical and Computer Modelling, 52 (2010), pp. $802-813$.

[9] A. Bellouquid and M. Delitala, Mathematical Modeling of Complex Biological Systems, Birkhäuser Boston, Inc., Boston, MA, 2006.

[10] W. Braun and K. Hepp, The Vlasov dynamics and its fluctuations in the $1 / N$ limit of interacting classical particles, Comm. Math. Phys., 56 (1977), pp. 101-113.

[11] M. Deaconu And N. Fournier, Probabilistic approach of some discrete and continuous coagulation equations with diffusion, Stochastic Process. Appl., 101 (2002), pp. 83-111.

[12] M. Deaconu, N. Fournier, And E. Tanré, Rate of convergence of a stochastic particle system for the Smoluchowski coagulation equation, Methodol. Comput. Appl. Probab., 5 (2003), pp. 131158.

[13] R. L. Dobrushin, Vlasov equations, Functional Analysis and Its Applications, 13 (1979), pp. $115-123$.

[14] Y. R. Domilovskiy, A. A. Lushnikov, and V. N. Piskurov, Monte Carlo simulation of coagulation processes, Dokl. Akad. Nauk SSSR Ser. Phys. Chem., (1978), pp. 108-110. 
[15] Dominik, C., Blum, J., Cuzzi, J.N., And Wurm, G., Protostar and planets V, University of Arizona Press, 2007, pp. 743-800.

[16] R. L. Drake, A general mathematical survey of the coagulation equation, in Topics in Current Aerosol Research (part2), International Reviews in Aerosol Physics and Chemistry, Pergamon Press, Oxford, 1972, pp. 203-376.

[17] _ The scalar transport equation of coalescence theory: Moments and kernels, J. Atmos. Sci., 29 (1972), pp. 537-547.

[18] A. Eibeck And W. Wagner, Approximative solution of the coagulation-fragmentation equation by stochastic particle systems, Stochastic Anal. Appl., 18 (2000), pp. 921-948.

[19] _ - An efficient stochastic algorithm for studying coagulation dynamics and gelation phenomena, SIAM J. Sci. Comput., 22 (2000), pp. 802-821 (electronic).

[20] _ Stochastic particle approximations for Smoluchoski's coagulation equation, Ann. Appl. Probab., 11 (2001), pp. 1137-1165.

[21] M. Escobedo And F. Pezzotti, In preparation.

[22] N. Fournier And J.-S. Giet, Convergence of the Marcus-Lushnikov process, Methodol. Comput. Appl. Probab., 6 (2004), pp. 219-231.

[23] S. K. Friedlander, Smoke, Dust, and Haze: Fundamentals of Aerosol Dynamics, Oxford University Press, 2000.

[24] A. L. Garcia, C. van den Broeck, M. Aertsens, and R. Serneels, A Monte Carlo simulation of coagulation, Physica A: Statistical Mechanics and its Applications, 143 (1987), pp. $535-546$.

[25] D. T. Gillespie, The Stochastic Coalescence Model for Cloud Droplet Growth., Journal of Atmospheric Sciences, 29 (1972), pp. 1496-1510.

[26] _ - A general method for numerically simulating the stochastic time evolution of coupled chemical reactions, Journal of Computational Physics, 22 (1976), pp. 403-434.

[27] S. Graffi, A. Martinez, And M. Pulvirenti, Mean-field approximation of quantum systems and classical limit, Math. Models Methods Appl. Sci., 13 (2003), pp. 59-73.

[28] F. Guiaş, A monte carlo approach to the Smoluchowski equations, Monte Carlo Meth. Appl, 3 (1997), pp. 313-326.

[29] — A direct simulation method for the coagulation-fragmentation equations with multiplicative coagulation kernels, Monte Carlo Methods Appl., 5 (1999), pp. 287-309.

[30] — Convergence properties of a stochastic model for coagulation-fragmentation processes with diffusion, Stochastic Analysis and Applications, 19 (2001), pp. 245-278. 
[31] — A stochastic approach for simulating spatially inhomogeneous coagulation dynamics in the gelation regime, Commun. Nonlinear Sci. Numer. Simul., 14 (2009), pp. 204-222.

[32] G. A. Jackson and S. E. Lochmann, effect of coagulation on nutrient and light limitation of an algal bloom, Limnology and Oceanography, 37 (1992), pp. 77-89.

[33] I. JEOn, Existence of gelling solutions for coagulation-fragmentation equations, Comm. Math. Phys., 194 (1998), pp. 541-567.

[34] _ S Spouge's conjecture on complete and instantaneous gelation, Journal of Statistical Physics, 96 (1999), pp. 1049-1070.

[35] M. LaChOwicZ, Stochastic semigroups and coagulation equations, Ukraïn. Mat. Zh., 57 (2005), pp. $770-777$.

[36] - Links between microscopic and macroscopic descriptions, in Multiscale problems in the life sciences, vol. 1940 of Lecture Notes in Math., Springer, Berlin, 2008, pp. 201-267.

[37] _ Individually-based Markov processes modeling nonlinear systems in mathematical biology, Nonlinear Anal. Real World Appl., 12 (2011), pp. 2396-2407.

[38] M. Lachowicz and M. Pulvirenti, A stochastic system of particles modelling the Euler equations, Arch. Rational Mech. Anal., 109 (1990), pp. 81-93.

[39] R. Lang And N. X. Xanh, Smoluchowski's theory of coagulation in colloids holds rigorously in the Boltzmann-Grad-limit, Probability Theory and Related Fields, 54 (1980), pp. 227-280.

[40] F. LeYvraz, Scaling theory and exactly solved models in the kinetics of irreversible aggregation, Physics Reports, 383 (2003), pp. $95-212$.

[41] A. A. Lushnikov, Coagulation in finite systems, Journal of Colloid and Interface Science, 65 (1978), pp. $276-285$.

[42] - Some new aspects of coagulation theory, Izv. Akad. Nauk SSSR Ser. Fiz. Atmosfer. i Okeana, 14 (1978), pp. $738-743$.

[43] A. H. Marcus, Stochastic coalescence, Technometrics, 10 (1968), pp. 133-143.

[44] C. M. Marques And M. E. Cates, Nonlinear thermodynamic relaxation in living polymer systems, J. Phys. II France, 1 (1991), pp. 489-492.

[45] Z. A. Melzak, A scalar transport equation, Trans. Amer. Math. Soc., 85 (1957), pp. 547-560.

[46] H. Neunzert, An introduction to the nonlinear Boltzmann-Vlasov equation, in Kinetic theories and the Boltzmann equation (Montecatini, 1981), vol. 1048 of Lecture Notes in Math., Springer, Berlin, 1984, pp. 60-110.

[47] J. R. NorRIS, Smoluchowski's coagulation equation: Uniqueness, nonuniqueness and a hydrodynamic limit for the stochastic coalescent., Ann. Appl. Probab., 9 (1999), pp. 78-109. 
[48] —, Brownian coagulation, Commun. Math. Sci., 2 (2004), pp. 93-101.

[49] F. Pezzotti, Mean-field limit and semiclassical approximation for quantum particle systems, Rend. Mat. Appl. (7), 29 (2009), pp. 223-340.

[50] D. Ruelle, Statistical mechanics: Rigorous results, W. A. Benjamin, Inc., New York-Amsterdam, 1969.

[51] K. K. Sabelfeld, S. V. Rogasinsky, A. A. Kolodko, and A. I. Levykin, Stochastic algorithms for solving Smolouchovsky coagulation equation and applications to aerosol growth simulation, Monte Carlo Methods Appl., 2 (1996), pp. 41-87.

[52] W. T. Scotт, Poisson Statistics in Distributions of Coalescing Droplets., J. Atmos. Sci., 24 (1967), pp. 221-225.

[53] J. Silk And T. Takahashi, A statistical model for the initial stellar mass function, Astrophys. J., 229 (1979), pp. 242-256.

[54] J. Silk And S. White, The development of structure in the expanding universe, Astrophys. J., 223 (1978), pp. L59-L62.

[55] M. V. Smoluchowski, Drei Vortrage uber Diffusion, Brownsche Bewegung und Koagulation von Kolloidteilchen, Zeitschrift fur Physik, 17 (1916), pp. 557-585.

[56] H. TANakA AND K. NakazaWa, Stochastic coagulation equation and validity of the statistical coagulation equation, J. Geomag. Geoelectr., 45 (1993), pp. 361 - 381.

[57] S. TAVARÉ, Line-of-descent and genealogical processes, and their applications in population genetics models, Theoret. Population Biol., 26 (1984), pp. 119-164.

[58] M. S. Turner And M. E. Cates, The relaxation spectrum of polymer length distributions, J. Phys. France, 51 (1990), pp. 307-316.

[59] W. Wagner, Explosion phenomena in stochastic coagulation-fragmentation models, Ann. Appl. Probab., 15 (2005), pp. 2081-2112.

[60] _ Post-gelation behavior of a spatial coagulation model, Electronic Journal of Probability, 11 (2006), pp. 893-933. 